\title{
New Exact Solutions and Modulation Instability for the Nonlinear (2+1)-Dimensional Davey-Stewartson System of Equation
}

\author{
Kwasi Boateng $(\mathbb{D}$, Weiguo Yang $(\mathbb{D}$, Wilson Osafo Apeanti, and David Yaro
}

Faculty of Science, Jiangsu University, Zhenjiang, Jiangsu Province 212013, China

Correspondence should be addressed to Kwasi Boateng; otalogboat@gmail.com

Received 28 May 2019; Accepted 22 July 2019; Published 6 August 2019

Guest Editor: Costica Morosanu

Copyright (c) 2019 Kwasi Boateng et al. This is an open access article distributed under the Creative Commons Attribution License, which permits unrestricted use, distribution, and reproduction in any medium, provided the original work is properly cited.

The Davey-Stewartson Equation (DSE) is an equation system that reflects the evolution in finite depth of soft nonlinear packets of water waves that move in one direction but in which the waves' amplitude is modulated in spatial directions. This paper uses the Generalized Elliptic Equation Rational Expansion (GEERE) technique to extract fresh exact solutions for the DSE. As a consequence, solutions with parameters of trigonometric, hyperbolic, and rational function are achieved. To display the physical characteristics of this model, the solutions obtained are graphically displayed. Modulation instability assessment of the outcomes acquired is also discussed and it demonstrates that all the solutions built are accurate and stable.

\section{Introduction}

Nonlinear partial differential equations (NLPDEs) are used in multiple study areas to define significant phenomena. Exact NLPDEs solutions play an important part in the research of physics, applied mathematics, and engineering, including solid state physics, fluid mechanics, population ecology, plasma physics, plasma waves, biology, optical fibres, propagation of shallow waves, heat flow, quantum mechanics, and wave propagation phenomena. Current studies are underway to find new techniques to extract traveling wave solution for NLPDEs. These techniques include the tanh-sech method [1], the Jacobi elliptic function method [2], the homotopy perturbation method [3], the homogeneous balance method [4], sine-cosine method $[5,6]$, the extended Weierstrass transformation method [7-10], the expansion method [11, 12], the Bäcklund transformation method [13], the inverse scattering method [14], modified extended mapping method [15], Darboux transformation [16], the extended tanh-coth method [17], Hirota bilinear method [18], modified extended direct algebraic method [19], lumped Galerkin method [20], and auxiliary equation method [21]. A lot of these methods are dependent on the type of problem and may or may not be suitable for other different problems [22-31].
The Davey-Stewartson Equation (DSE) [32] is an equation system that reflects the evolution in finite depth of soft nonlinear packets of water waves traveling in one direction but in which the amplitude of waves is modulated in spatial directions. This equation also defines long wave-short wave resonances and the development of a 3-dimensional wave packet on finite depth water [33,34]. A few solutions have been acquired for this equation [35-38]. The DSE, a twodimensional system that coexists with both short waves and long waves, and a precise representation of two-dimensional modulation of nonlinear waves should require both short wave and long wave modes. A pair of coupled nonlinear DSE in two dependent variables can be decreased to the (1+1)-dimensional nonlinear Schrödinger (NLS) equation by carrying out a suitable dimensional reduction. A number of analytical methods have been created to be used for NLPDEs such as the DSE, which in recent years have specific types of solutions, such as growing and decaying solutions [3941], dromions, breathers, instantaneous, propagating, and regular wave patterns, and fluid flow and heat transfer over a stretching or shrinking sheet in a porous medium [34, 42, 43].

The Generalized Elliptic Equation Rational Expansion (GEERE) technique developed by Wan, Song, Yin, and 
Zhang [44] is applied in this paper. This technique is a very strong technique that can be used to obtain several particular solutions including rational formal regular triangular, Weierstrass doubly regular, rational formal solitary wave, and rational formal Jacobi solutions [44]. In addition, the modulation instability assessment of the solutions obtained will be explored. Modulation instability is universal, and the basis for the formation of soliton solutions results from the interaction between dispersion and nonlinearity in the spatial and time domain [45-47]. It is therefore of interest to derive explicit DSE equation solutions using the GEERE method. This paper is structured as follows: in Section 2 new soliton solutions are built for DSE. DSE assessment of modulation instability is shown in Section 3. Section 4 also presents the outcomes and discussion. Finally, in Section 5, the conclusion is provided.

\section{Nonlinear (2+1)-Dimensional Davey- Stewartson Equation}

The Davey-Stewartson system [32] is of the form

$$
\begin{array}{r}
i \frac{\partial Z}{\partial t}+\frac{1}{2} \alpha^{2}\left(\frac{\partial^{2} Z}{\partial x^{2}}+\alpha^{2} \frac{\partial^{2} Z}{\partial y^{2}}\right)-\nu|Z|^{2} Z+Z \frac{\partial Q}{\partial x}=0 \\
\frac{\partial^{2} Q}{\partial x^{2}}-\alpha^{2} \frac{\partial^{2} Q}{\partial y^{2}}-2 \nu \frac{\partial|Z|^{2}}{\partial x}=0 .
\end{array}
$$

Here $z(x, y, t)$ is a complex value function, $x$ is the dimensionless variable, $y$ is the propagation coordinate, $t$ is the time, $v= \pm 1$, and $\alpha^{2}= \pm 1$. The case where $\alpha=1$ is called the DSE I equation, while that where $\alpha=i$ is the DSE II. The $v$ parameter denotes the focusing or defocusing case. The DSE has the following types of soliton solutions: conventional line, algebraic, periodic, and lattice solution. Conventional line soliton has a structure that is essentially one-dimensional. The solitons of periodic, algebraic, and lattice have a structure of two dimensions. The DSE I and II are common examples of two-dimensional integrable equations that emerge as a higher-dimensional generalization of the nonlinear Schrödinger equation (NLSE). DSE has several applications that include the description of gravitycapillarity surface wave packets within the shallow water boundary. Several strong techniques have been created to obtain explicit solutions for (1), such as the technique of homotopy analysis [48], the sine-cosine method [49], and the technique of variational iteration [50]. To obtain fresh soliton solutions for (1) using the following traveling wave equation, we apply the widely discussed GEERE technique in [44]:

$$
\begin{aligned}
z(x, y, t) & =Z(\xi) e^{i \gamma}, \\
q(x, y, t) & =Q(\xi), \\
\xi & =x+y-c t, \\
\gamma & =k_{1} x+k_{2} y+k_{3} t,
\end{aligned}
$$

where $c, k_{1}, k_{2}$, and $k_{3}$ are real constants. Substituting (2) into (1) gives the real and imaginary part:

$$
\begin{aligned}
c & =\alpha^{2}\left(k_{1}+\alpha^{2} k_{2}\right), \\
\alpha^{2}\left(1+\alpha^{2}\right) Z^{\prime \prime}(\xi)+2 \nu Z^{3}(\xi) & \\
& -2\left[\left(Q^{\prime}(\xi)+k_{3}\right)+\alpha^{2}\left(k_{1}^{2}+\alpha^{2} k_{2}^{2}\right)\right] Z(\xi), \\
& \left(1-\alpha^{2}\right) Q^{\prime \prime}(\xi)-2 \nu\left(Z^{2}(\xi)\right)^{\prime}=0 .
\end{aligned}
$$

Integrating (5) with respect to $\xi$ and setting the constant of integration to zero, we have

$$
Q^{\prime}(\xi)=\frac{2 \nu}{1-\alpha^{2}} Z^{2}(\xi)
$$

Putting (6) into (4) gives

$$
\begin{aligned}
& \alpha^{2}\left(\alpha^{4}-1\right) Z^{\prime \prime}(\xi)+2 \nu\left(\alpha^{2}+1\right) Z^{3}(\xi) \\
& \quad-\left(\alpha^{2}-1\right)\left[2 k_{3}+\alpha^{2}\left(k_{1}^{2}+\alpha^{2} k_{2}^{2}\right)\right] Z(\xi)=0 .
\end{aligned}
$$

Introducing a new ansatz of finite rational expansion in the form:

$$
Z(\xi)=a_{0}+\sum_{i=1}^{N} \frac{a_{i} \phi^{i}(\xi)+b_{i} \phi^{i-1}(\xi) \phi^{\prime}(\xi)}{(\mu \phi(\xi)+1)^{i}}
$$

the new parameter $\phi=\phi(\xi)$ and

$$
\phi^{\prime}=\sqrt{\sum_{j=0}^{4} l_{j} \phi^{j}}
$$

where $l_{j}, a_{0}, a_{i}$, and $b_{i}(j=0,1,2, \ldots ; i=1,2,3, \ldots, N)$ are constant real values to be computed. Using homogeneous balance principle by balancing the highest nonlinear terms and the highest order partial derivative terms in (7) we have $N=1$; hence

$$
Z(\xi)=a_{0}+\frac{a_{1} \phi(\xi)+b_{1} \phi^{\prime}(\xi)}{\mu \phi(\xi)+1}
$$

putting (10) and (9) in (7) we have a system of algebraic equations in parameters, $l_{1}, l_{2}, l_{3}, l_{4}, a_{0}, a_{0}, a_{1}, b_{1}, \mu$. The system of algebraic equations after solving gives the following family of solutions with respect to different cases.

Case 1. $l_{4}=0, l_{3}=0$.

Family 1.1. $a_{0}= \pm \sqrt{-\left(\alpha^{2}\left(\alpha^{2}-1\right) l_{2}\right) / \kappa} / \sqrt{2}, a_{1}=a_{1}, b_{1}=a_{1}$, $k_{3}=-(1 / 4) \alpha^{2}\left(2 k_{2}^{2} \sigma^{2}+2 k_{1}^{2}+\alpha^{2} l_{2}+l_{2}\right), \mu=0$ 


$$
\begin{aligned}
& Z_{11}(\xi)= \pm \frac{\sqrt{-\left(\alpha^{2}\left(\alpha^{2}-1\right) l_{2}\right) / \kappa}}{\sqrt{2}}+a_{1}\left(e^{\sqrt{l_{2(-c t+x+y)}}}-\frac{l_{1}}{2 l_{2}}\right) \\
& \pm a_{1} \sqrt{l_{2}\left(e^{\sqrt{l_{2}(-c t+x+y)}}-\frac{l_{1}}{2 l_{2}}\right)^{2}+l_{1}\left(e^{\sqrt{l_{2}(-c t+x+y)}}-\frac{l_{1}}{2 l_{2}}\right)+l_{0}} \\
& \times \exp \left[i\left(k_{1} x+k_{2} y-\frac{1}{4} \alpha^{2} t\left(2 k_{2}^{2} \sigma^{2}+2 k_{1}^{2}+\alpha^{2} l_{2}+l_{2}\right)\right)\right] \text {, } \\
& Q_{11}(\xi)=\frac{2 v}{3\left(1-\alpha^{2}\right)},\left(Z_{11}(\xi)\right)^{3}, \\
& l_{0}=\frac{l_{1}^{2}}{4 l_{2}} \\
& l_{2}>0 \text {. } \\
& Z_{12}(\xi)= \pm \frac{\sqrt{-\left(\alpha^{2}\left(\alpha^{2}-1\right) l_{2}\right) / \kappa}}{\sqrt{2}} \\
& +\frac{a_{1}\left(\left(l_{2} \sqrt{\left.l_{1}^{2}\left(\sin ^{2}\left(\sqrt{l_{2}(-(-c t+x+y)}\right)\right)-1\right) / l_{2}}\right)+l_{1}\left(\sin \left(\sqrt{l_{2}(-(-c t+x+y))}\right)-1\right)\right)}{2 l_{2}} \\
& \times \exp \left[i\left(k_{1} x+k_{2} y-\frac{1}{4} \alpha^{2} t\left(2 k_{2}^{2} \sigma^{2}+2 k_{1}^{2}+\alpha^{2} l_{2}+l_{2}\right)\right)\right], \\
& Q_{12}(\xi)=\frac{2 \nu}{3\left(1-\alpha^{2}\right)}\left(Z_{12}(\xi)\right)^{3}, \\
& l_{0}=0, \\
& l_{2}<0 \text {. } \\
& Z_{13}(\xi)= \pm \frac{\sqrt{-\alpha^{2}\left(\alpha^{2}-1\right) l_{2} / \kappa}}{\sqrt{2}} \\
& +\frac{a_{1}\left(l_{2} \sqrt{\left.\left(l_{1}^{2}\left(\sin ^{2} h\left(\sqrt{l_{2}((-c t+x+y)}\right)\right)-1\right) / l_{2}\right)+4 l_{0}}+l_{1}\left(\sinh \left(\sqrt{l_{2}((-c t+x+y))}\right)-1\right)\right)}{2 l_{2}} \\
& \times \exp \left[i\left(k_{1} x+k_{2} y-\frac{1}{4} \alpha^{2} t\left(2 k_{2}^{2} \sigma^{2}+2 k_{1}^{2}+\alpha^{2} l_{2}+l_{2}\right)\right)\right], \\
& Q_{13}(\xi)=\frac{2 v}{3\left(1-\alpha^{2}\right)}\left(Z_{13}(\xi)\right)^{3} \\
& l_{0}=0 \text {, } \\
& l_{2}>0 \text {. }
\end{aligned}
$$

Family 1.2. $a_{0}=0, a_{1}=a_{1}, b_{1}=$ $2 \sqrt{\left(\alpha^{2}\left(\alpha^{2}-1\right)(-\kappa) l_{2}^{2}\left(l_{1}^{2}-4 l_{0} l_{2}\right)^{2}\right)} /\left(\kappa l_{1}^{3}-4 \kappa l_{0} l_{1} l_{2}\right) \pm a_{1}$, $k_{3}=(1 / 2) \alpha^{2}\left(2 l_{2} \alpha^{2}+2 l_{2}+k_{2}^{2} \sigma^{2}+k_{1}^{2}\right), \mu=0$ 


$$
\begin{aligned}
& Z_{14}(\xi)=a_{1}\left(e^{\sqrt{l_{2}(-c t+x+y)}}-\frac{l_{1}}{2 l_{2}}\right) \pm a_{1}+\left(\frac{l_{1}}{2 l_{2}\left(e^{\sqrt{l_{2}(-c t+x+y)}}-l_{1} / 2 l_{2}\right)+l_{1}}\right) \frac{2}{\kappa l_{1}^{3}-4 \kappa l_{0} l_{1} l_{2}} \\
& \times\left[\sqrt{l_{2}\left(e^{\sqrt{l_{2}(-c t+x+y)}}-\frac{l_{1}}{2 l_{2}}\right)^{2}+l_{1}\left(e^{\sqrt{l_{2}(-c t+x+y)}}-\frac{l_{1}}{2 l_{2}}\right)+l_{0}} \sqrt{-\alpha^{2}\left(\alpha^{2}-1\right) \kappa l_{2}^{2}\left(l_{1}^{2}-4 l_{0} l_{2}\right)^{2}}\right] \\
& \times \exp \left[i\left(k_{1} x+k_{2} y+\frac{1}{2} \alpha^{2}\left(2 l_{2} \alpha^{2}+2 l_{2}+k_{2}^{2} \sigma^{2}+k_{1}^{2}\right)\right)\right] \text {, } \\
& Q_{14}(\xi)=\frac{2 \nu}{3\left(1-\alpha^{2}\right)}\left(Z_{14}(\xi)\right)^{3} \\
& l_{0}=\frac{l_{1}^{2}}{4 l_{2}} \\
& l_{2}>0 \text {. } \\
& Z_{15}(\xi)=\frac{\csc \left(\sqrt{l_{2}(c t-x-y)}\right)}{2 \kappa l_{1}^{3} l_{2}}\left(a_{1} \kappa l_{1}^{3}\left(l_{1}\left(\sin \left(\sqrt{l_{2}(c t-x-y)}\right)-1\right)-2 l_{2}\right)+2 l_{2} \sqrt{-\alpha^{2}\left(\alpha^{2}-1\right) \kappa l_{1}^{4} l_{2}^{2}}\right. \\
& \left.\times \sqrt{-\frac{l_{1}^{2} \cos ^{2}\left(\sqrt{l_{2}(c t-x-y)}\right)}{l_{2}}}\right) \times \exp \left[i\left(k_{1} x+k_{2} y+\frac{1}{2} \alpha^{2}\left(2 l_{2} \alpha^{2}+2 l_{2}+k_{2}^{2} \sigma^{2}+k_{1}^{2}\right)\right)\right], \\
& Q_{15}(\xi)=\frac{2 \nu}{3\left(1-\alpha^{2}\right)}\left(Z_{15}(\xi)\right)^{3}, \\
& l_{0}=0 \text {, } \\
& l_{2}<0 \text {. } \\
& Z_{16}(\xi)=\frac{\operatorname{csch}\left(\sqrt{-l_{2}(c t-x-y)}\right)}{2 \kappa l_{1}^{3} l_{2}}\left[a_{1} \kappa l_{1}^{3}\left(l_{1}\left(\sinh \left(\sqrt{-l_{2}(c t-x-y)}\right)-1\right)-2 l_{2}\right)+2 l_{2} \sqrt{-\alpha^{2}\left(\alpha^{2}-1\right) \kappa l_{1}^{4} l_{2}^{2}}\right.
\end{aligned}
$$

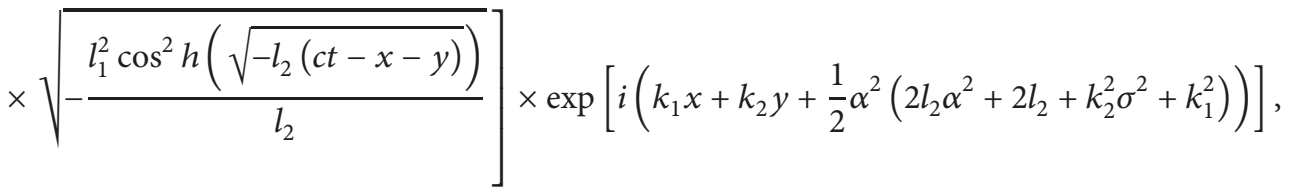

$$
\begin{aligned}
& Q_{16}(\xi)=\frac{2 \nu}{3\left(1-\alpha^{2}\right)}\left(Z_{16}(\xi)\right)^{3} \\
& l_{0}=0 \text {, } \\
& l_{2}<0 \text {. }
\end{aligned}
$$

Case 2. $l_{1}=0, l_{3}=0$. 
Family 2.1. $a_{0}=-\sqrt{-\alpha^{2}\left(\alpha^{2}-1\right) l_{2} / \kappa} / \sqrt{2}, a_{1}=a_{1}, b_{1}=a_{1}$, $k_{3}=-(1 / 4) \alpha^{2} t\left(2 k_{2}^{2} \sigma^{2}+2 k_{1}^{2}+\alpha^{2} l_{2}+l_{2}\right), \mu=0$

$$
\begin{aligned}
& Z_{21}(\xi)=a_{1} \sqrt{\kappa} \sqrt{l_{0}} \sqrt{l_{4}^{2} l_{2}^{3} \tan ^{2}\left(\frac{\sqrt{l_{2}}(-c t+x+y)}{\sqrt{2}}\right)\left(l_{2} l_{4}^{3} \tan ^{2}\left(\frac{\sqrt{l_{2}}(-c t+x+y)}{\sqrt{2}}\right)+4\right)+16 l_{0}} \\
& \quad+2 l_{2} l_{4} \sqrt{l_{0}} a_{1} \sqrt{\kappa} \tan \left(\frac{\sqrt{l_{2}}(-c t+x+y)}{\sqrt{2}}\right)-2 \sqrt{l_{0}} \sqrt{-\alpha^{2}\left(\alpha^{2}-1\right) l_{2}^{2}} \\
& \quad \times \exp \left[i\left(k_{1} x+k_{2} y-\frac{1}{4} \alpha^{2} t\left(2 k_{2}^{2} \sigma^{2}+2 k_{1}^{2}+\alpha^{2} l_{2}+l_{2}\right)\right)\right], \\
& Q_{21}(\xi)=\frac{2 v}{3\left(1-\alpha^{2}\right)}\left(Z_{21} 3(\xi)\right)^{3}, \\
& l_{0}=\frac{l_{1}^{2}}{4 l_{2}}, \\
& l_{2}>0, \\
& l_{4}<0 . \\
& Z_{22}(\xi)=a_{1} \sqrt{\kappa} \sqrt{l_{0}} \sqrt{l_{4}^{2} l_{2}^{3} \tan ^{2} h\left(\frac{\sqrt{l_{2}}(-c t+x+y)}{\sqrt{2}}\right)\left(l_{2} l_{4}^{3} \tan ^{2} h\left(\frac{\sqrt{l_{2}}(-c t+x+y)}{\sqrt{2}}\right)+4\right)+16 l_{0}} \\
& \quad+2 l_{2} l_{4} \sqrt{l_{0}} a_{1} \sqrt{\kappa} \tanh \left(\frac{\sqrt{l_{2}}(-c t+x+y)}{\sqrt{2}}\right)-2 \sqrt{l_{0}} \sqrt{-\alpha^{2}\left(\alpha^{2}-1\right) l_{2}^{2}} \\
& \quad \times \exp \left[i\left(k_{1} x+k_{2} y-\frac{1}{4} \alpha^{2} t\left(2 k_{2}^{2} \sigma^{2}+2 k_{1}^{2}+\alpha^{2} l_{2}+l_{2}\right)\right)\right], \\
& Q_{22}(\xi)=\frac{2 v}{3\left(1-\alpha^{2}\right)}\left(Z_{22} 3(\xi)\right)^{3}, \\
& l_{0}=\frac{l_{1}^{2}}{4 l_{2}}, \\
& l_{4}>0 .
\end{aligned}
$$

Family 2.2. $a_{0}=0, a_{1}=a_{1}, b_{1}=\left(\left(\sqrt{\alpha^{4} \kappa l_{0} l_{2}-\alpha^{2} \kappa l_{0} l_{2}}-\right.\right.$

$\left.\left.2 a_{1} \kappa l_{0}\right) / 2 \kappa l_{0}\right), k_{3}=-(1 / 4) \alpha^{2} t\left(2 k_{2}^{2} \sigma^{2}+2 k_{1}^{2}+\alpha^{2} l_{2}+l_{2}\right)$, $\mu=-i \sqrt{l_{2}} / \sqrt{l_{0}}$

$$
\begin{aligned}
& Z_{23}(\xi)=\frac{\kappa l_{0} 16 \sqrt{l_{0}}}{16 \sqrt{l_{0}}-8 i \sqrt{l_{2}} l_{2} l_{4} \tan \left(\sqrt{l_{2}}(-c t+x+y) / \sqrt{2}\right)}\left[\left(\sqrt{\alpha^{2}\left(\alpha^{2}-1\right) \kappa l_{0} l_{2}}-2 a_{1} \kappa l_{0}\right)\right. \\
& \quad \times \sqrt{l_{4}^{2} l_{2}^{3} \tan ^{2}\left(\frac{\sqrt{l_{2}}(-c t+x+y)}{\sqrt{2}}\right)\left(l_{2} l_{4}^{3} \tan ^{2}\left(\frac{\sqrt{l_{2}}(-c t+x+y)}{\sqrt{2}}\right)+4\right)+16 l_{0}} \\
& \left.\quad+4 a_{1} l_{2} l_{4} \kappa l_{0} \tan \left(\frac{\sqrt{l_{2}}(-c t+x+y)}{\sqrt{2}}\right)\right] \times \exp \left[i\left(k_{1} x+k_{2} y-\frac{1}{4} \alpha^{2} t\left(2 k_{2}^{2} \sigma^{2}+2 k_{1}^{2}+\alpha^{2} l_{2}+l_{2}\right)\right)\right],
\end{aligned}
$$




$$
\begin{aligned}
& Q_{23}(\xi)=\frac{2 \nu}{3\left(1-\alpha^{2}\right)}\left(Z_{23} 3(\xi)\right)^{3}, \\
& l_{0}=\frac{l_{1}^{2}}{4 l_{2}} \\
& l_{2}>0 \text {, } \\
& l_{4}>0 \text {. } \\
& Z_{24}(\xi)=\frac{\kappa l_{0} 16 \sqrt{l_{0}}}{16 \sqrt{l_{0}}-8 i \sqrt{l_{2}} l_{2} l_{4} \tanh \left(\sqrt{-l_{2}}(-c t+x+y) / \sqrt{2}\right)}\left[\left(\sqrt{\alpha^{2}\left(\alpha^{2}-1\right) \kappa l_{0} l_{2}}-2 a_{1} \kappa l_{0}\right)\right. \\
& \times \sqrt{l_{4}^{2} l_{2}^{3} \tan ^{2}\left(\frac{\sqrt{-l_{2}}(-c t+x+y)}{\sqrt{2}}\right)\left(l_{2} l_{4}^{3} \tanh ^{2}\left(\frac{\sqrt{-l_{2}}(-c t+x+y)}{\sqrt{2}}\right)+4\right)+16 l_{0}} \\
& \left.+4 a_{1} l_{2} l_{4} \kappa l_{0} \tan \left(\frac{\sqrt{-l_{2}}(-c t+x+y)}{\sqrt{2}}\right)\right] \times \exp \left[i\left(k_{1} x+k_{2} y-\frac{1}{4} \alpha^{2} t\left(2 k_{2}^{2} \sigma^{2}+2 k_{1}^{2}+\alpha^{2} l_{2}+l_{2}\right)\right)\right] \\
& Q_{24}(\xi)=\frac{2 \nu}{3\left(1-\alpha^{2}\right)}\left(Z_{24} 3(\xi)\right)^{3} \\
& l_{0}=\frac{l_{1}^{2}}{4 l_{2}} \\
& l_{2}<0 \text {, } \\
& l_{4}>0 \text {. }
\end{aligned}
$$

Case 3. $l_{3}=0, l_{4}=0, l_{0}=0$.

Family 3.1. $a_{0}=0, a_{1}=a_{1}, b_{1}=\left(\sqrt{\kappa h_{2}^{2} \alpha^{2}-\kappa l_{2}^{2} \alpha^{4}}-\right.$ $\left.2 a_{1} \kappa l_{1}\right) / 2 \kappa l_{1}, k_{3}=-(1 / 4) \sigma^{2}\left(l_{2} \alpha^{2}+l_{2}+2 k_{2}^{2} \alpha^{2}+2 k_{1}^{2}\right), \mu=l_{2} / l_{1}$

$$
\begin{aligned}
Z_{31}(\xi)= & \frac{\left.2 a_{1} \kappa l_{1}\left(l_{1}\left(\sin \left(\sqrt{l_{2}(-(-c t+x+y))}\right)-1\right)-l_{2} \sqrt{\left(l_{1}^{2}\left(\sin ^{2}\left(\sqrt{l_{2}(-(-c t+x+y))}\right)-1\right)\right.}\right) / l_{2}\right)}{2 \kappa l_{1} l_{2}\left(\sin \left(\sqrt{l_{2}(-(-c t+x+y))}\right)+1\right)} \\
& +\frac{2 \kappa l_{1} l_{2}\left(\sin \left(\sqrt{l_{2}(-(-c t+x+y))}\right)+1\right)+l_{2} \sqrt{-\alpha^{2}\left(\alpha^{2}-1\right) \kappa l_{2}^{2}} \sqrt{l_{1}^{2}\left(\sin ^{2}\left(\sqrt{l_{2}(-(-c t+x+y))}-1\right) / l_{2}\right.}}{2 \kappa l_{1} l_{2}\left(\sin \left(\sqrt{l_{2}(-(-c t+x+y))}\right)+1\right)}
\end{aligned}
$$$$
\times \exp \left[i\left(k_{1} x+k_{2} y-\frac{1}{4} \alpha^{2} t\left(2 k_{2}^{2} \sigma^{2}+2 k_{1}^{2}+\alpha^{2} l_{2}+l_{2}\right)\right)\right],
$$

$$
Q_{31}(\xi)=\frac{2 \nu}{3\left(1-\alpha^{2}\right)}\left(Z_{31} 3(\xi)\right)^{3}
$$$$
l_{2}<0 \text {. }
$$ 


$$
\begin{aligned}
Z_{32}(\xi)= & \frac{2 a_{1} \kappa l_{1}\left(l_{1}\left(\sinh \left(\sqrt{l_{2}(-c t+x+y)}\right)-1\right)-l_{2} \sqrt{l_{1}^{2}\left(\sin ^{2} h\left(\sqrt{l_{2}(-c t+x+y)}\right)-1\right) / l_{2}}\right)}{2 \kappa l_{1} l_{2}\left(\sinh \left(\sqrt{l_{2}(-c t+x+y)}\right)+1\right)} \\
& +\frac{2 \kappa l_{1} l_{2}\left(\sinh \left(\sqrt{l_{2}(-c t+x+y)}\right)+1\right)+l_{2} \sqrt{-\alpha^{2}\left(\alpha^{2}-1\right) \kappa l_{2}^{2}} \sqrt{l_{1}^{2}\left(\sin ^{2} h\left(\sqrt{l_{2}(-c t+x+y)}\right)-1\right) / l_{2}}}{2 \kappa l_{1} l_{2}\left(\sinh \left(\sqrt{l_{2}(-c t+x+y)}\right)+1\right)} \\
& \times \exp \left[i\left(k_{1} x+k_{2} y-\frac{1}{4} \alpha^{2} t\left(2 k_{2}^{2} \sigma^{2}+2 k_{1}^{2}+\alpha^{2} l_{2}+l_{2}\right)\right)\right], \\
Q_{32}(\xi)= & \frac{2 \nu}{3\left(1-\alpha^{2}\right)}\left(Z_{32} 3(\xi)\right)^{3}, \\
l_{2}> & 0 .
\end{aligned}
$$

Family 3.2. $a_{0}=0, a_{1}=a_{1}, b_{1}=\left(2 \sqrt{\kappa h_{2}^{2} \alpha^{2}-\kappa l_{2}^{2} \alpha^{4}}-\right.$ $\left.a_{1} \kappa l_{1}\right) / \kappa l_{1}, k_{3}=-(1 / 2) \alpha^{2}\left(2 l_{2} \alpha^{2}+2 l_{2}+k_{2}^{2} \alpha^{2}+k_{1}^{2}\right), \mu=2 l_{2} / l_{1}$

$$
\begin{aligned}
& Z_{33}(\xi)=\frac{1}{2 \kappa l_{1} l_{2}}\left(\csc \left(\sqrt{l_{2}(-(-c t+x+y))}\right)\right. \\
& \cdot\left[a _ { 1 } \kappa l _ { 1 } \left[l_{1}\left(\sin \left(\sqrt{l_{2}(-(-c t+x+y))}\right)-1\right)\right.\right. \\
& \left.-l_{2} \sqrt{\frac{l_{1}^{2}\left(\sin ^{2}\left(\sqrt{l_{2}(-(-c t+x+y))}\right)-1\right)}{l_{2}}}\right] \\
& +2 l_{2} \sqrt{-\alpha^{2}\left(\alpha^{2}-1\right) \kappa l_{2}^{2}} \\
& \left.\times \sqrt{\left.\frac{l_{1}^{2}\left(\sin ^{2}\left(\sqrt{l_{2}(-(-c t+x+y))}\right)-1\right)}{l_{2}}\right]}\right) \\
& \times \exp \left[i \left(k_{1} x+k_{2} y-\frac{1}{2} \alpha^{2}\left(2 l_{2} \alpha^{2}+2 l_{2}+k_{2}^{2} \alpha^{2}\right.\right.\right. \\
& \left.\left.\left.+k_{1}^{2}\right)\right)\right] \text {, } \\
& Q_{33}(\xi)=\frac{2 v}{3\left(1-\alpha^{2}\right)}\left(Z_{33} 3(\xi)\right)^{3}, \\
& l_{2}<0
\end{aligned}
$$

$$
\begin{aligned}
& Z_{34}(\xi)=\frac{1}{2 \kappa l_{1} l_{2}}\left(\operatorname{csch}\left(\sqrt{l_{2}(-c t+x+y)}\right)\right. \\
& {\left[a _ { 1 } \kappa l _ { 1 } \left[l_{1}\left(\sinh \left(\sqrt{l_{2}(-c t+x+y)}\right)-1\right)\right.\right.} \\
& \left.-l_{2} \sqrt{\frac{l_{1}^{2}\left(\sin ^{2} h\left(\sqrt{l_{2}(-c t+x+y)}\right)-1\right)}{l_{2}}}\right] \\
& +2 l_{2} \sqrt{-\alpha^{2}\left(\alpha^{2}-1\right) \kappa l_{2}^{2}} \\
& \left.\left.\times \sqrt{\frac{l_{1}^{2}\left(\sin ^{2} h\left(\sqrt{l_{2}(-c t+x+y)}\right)-1\right)}{l_{2}}}\right]\right) \\
& \times \exp \left[i \left(k_{1} x+k_{2} y-\frac{1}{2} \alpha^{2}\left(2 l_{2} \alpha^{2}+2 l_{2}+k_{2}^{2} \alpha^{2}\right.\right.\right. \\
& \left.\left.\left.+k_{1}^{2}\right)\right)\right] \\
& Q_{34}(\xi)=\frac{2 \nu}{3\left(1-\alpha^{2}\right)}\left(Z_{34} 3(\xi)\right)^{3}, \\
& l_{2}>0 \text {. }
\end{aligned}
$$

Case 4. $l_{0}=0, l_{1}=0, l_{4}=0$.

Family 4.1. $a_{0}=0, a_{1}=a_{1}, b_{1}=\left(\left(\left(\sqrt{\alpha^{2} \kappa l_{3}^{2}-\alpha^{4} \kappa l_{3}^{2}} / l_{2}\right)-\right.\right.$ $\left.\left.2 a_{1} \kappa\right) / 2 \kappa\right), k_{3}=-(1 / 2) \alpha^{2} t\left(k_{2} \alpha^{2}+l_{2}-k_{2}^{2} \alpha^{2}-l_{1}^{2}\right), \mu=l_{3} / l_{2}$ 


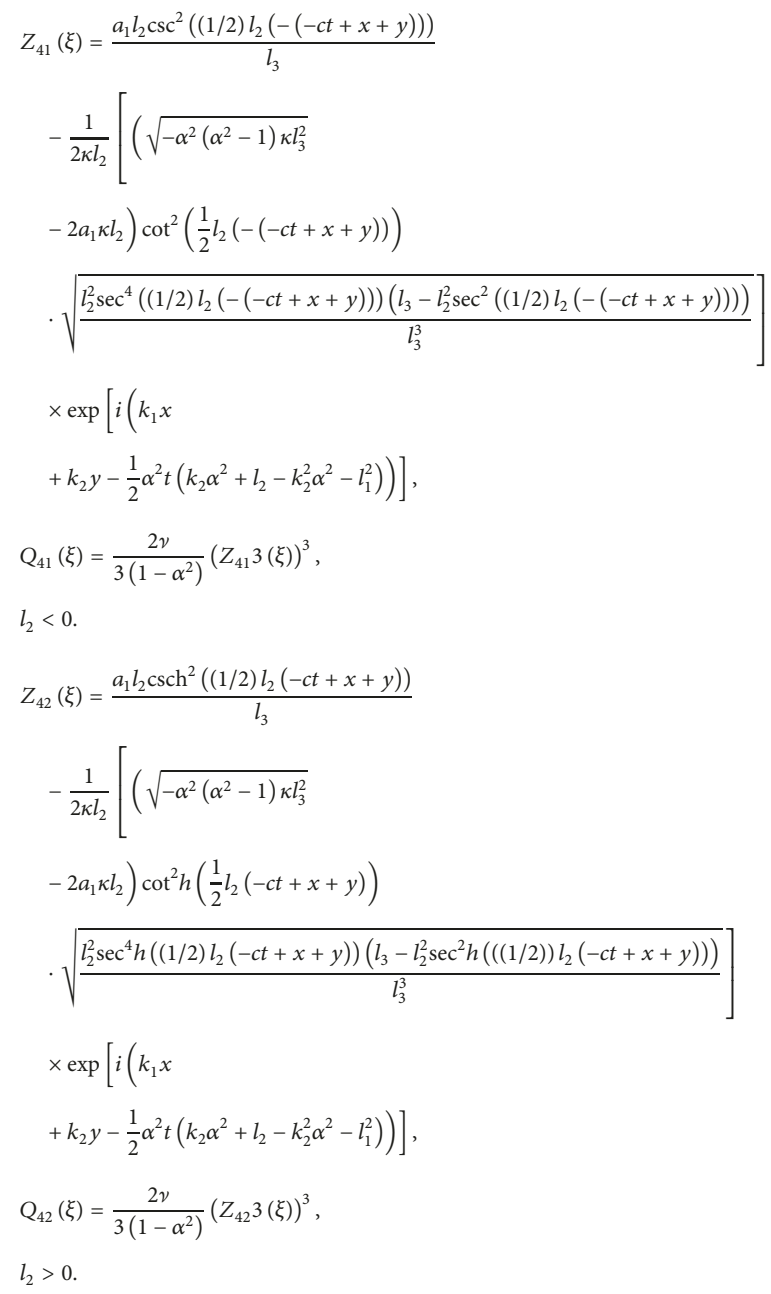

Family 4.2. $a_{0}=0, a_{1}=a_{1}, b_{1}=\left(-2 a_{1} \sqrt{\kappa}+i \sqrt{2} \mu\right) / 2 \sqrt{\kappa}$, $k_{3}=(1 / 2)\left(k_{1}^{2}-k_{2}^{2}\right), \mu=0$

$Z_{43}(\xi)$

$$
=\frac{1}{2 \sqrt{\kappa}}[i(\sqrt{2} \mu
$$

$Z_{51}(\xi)$

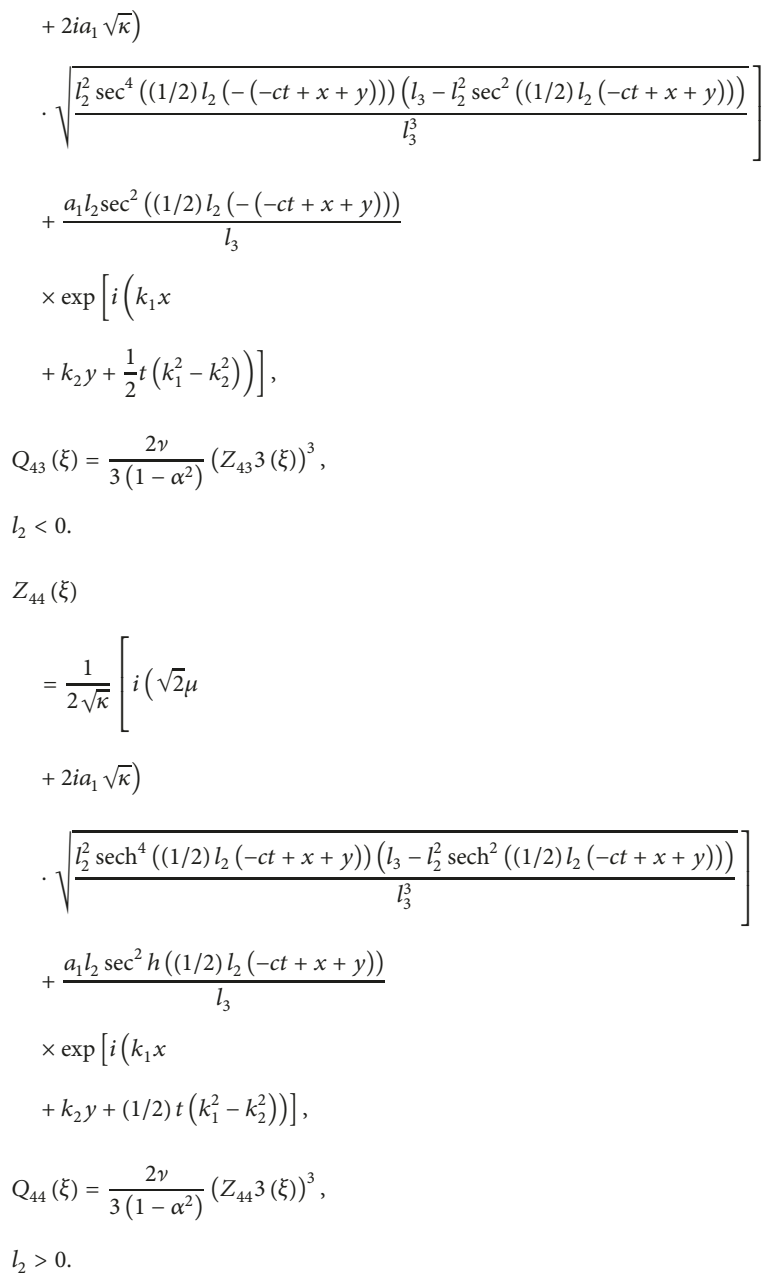

Case 5. $l_{0}=0, l_{1}=0$.

Family 5.1. $a_{0}=0, a_{1}=a_{1}, b_{1}=\left(a_{1}(-\sqrt{\kappa}) l_{3}-4 i \sqrt{2} l_{4}\right) / \sqrt{\kappa} l_{3}$, $k_{3}=(1 / 2)\left(k_{1}^{2}-k_{2}^{2}\right), \mu=4 l_{4} / l_{3}$

$$
\begin{aligned}
& \quad=\frac{1}{\left(4 l_{2} l_{4} \sec ^{2}\left(-\sqrt{l_{2}} \xi / 2\right) / l_{3}\left(2 \sqrt{-l_{2} l_{4}} \tan \left((1 / 2) \sqrt{-l_{2}} \xi\right)+l_{3}\right)\right)+1}\left[\frac{a_{1} l_{2} \sec ^{2}\left(\sqrt{-l_{2}} \xi / 2\right)}{2 \sqrt{-l_{2} l_{4}} \tan \left((1 / 2) \sqrt{-l_{2}} \xi\right)+l_{3}}+\left(\frac{\left(a_{1}(-\sqrt{\kappa}) l_{3}-4 i \sqrt{2} l_{4}\right)}{\sqrt{\kappa} l_{3}} \times\left[\frac{l_{4} l_{2}^{4} \sec ^{8}\left(\sqrt{-l_{2}} \xi / 2\right)}{\left(2 \sqrt{-l_{2} l_{4}} \tan \left((1 / 2) \sqrt{-l_{2}} \xi\right)+l_{3}\right)^{4}}\right.\right.\right. \\
& +\frac{l_{3} l_{2}^{3} \sec ^{6}\left(\sqrt{-l_{2}} \xi / 2\right)}{\left(2 \sqrt{-l_{2} l_{4}} \tan \left((1 / 2) \sqrt{-l_{2}} \xi\right)+l_{3}\right)^{3}} \\
& \left.\left.\left.+\frac{l_{2}^{3} \sec ^{4}\left(\sqrt{-l_{2}} \xi / 2\right)}{\left(2 \sqrt{-l_{2} l_{4}} \tan \left((1 / 2) \sqrt{-l_{2}} \xi\right)+l_{3}\right)^{2}}\right]^{1 / 2}\right)\right] \\
& \quad \times \exp \left[i \left(k_{1} x\right.\right. \\
& \left.\left.\quad+k_{2} y+\frac{1}{2} t\left(k_{1}^{2}-k_{2}^{2}\right)\right)\right] \\
& Q_{51}(\xi)=\frac{2 v}{3\left(1-\alpha^{2}\right)}\left(Z_{51} 3(\xi)\right)^{3} \\
& l_{2}<0 .
\end{aligned}
$$




$$
\begin{aligned}
& Z_{52}(\xi) \\
& \quad=\frac{1}{\left(4 l_{2} l_{4} \sec ^{2}\left(\sqrt{l_{2}} \xi / 2\right) / l_{3}\left(2 \sqrt{l_{2} l_{4}} \tan \left((1 / 2) \sqrt{l_{2}} \xi\right)+l_{3}\right)\right)+1}\left[\frac{a_{1} l_{2} \sec ^{2}\left(\sqrt{l_{2}} \xi / 2\right)}{2 \sqrt{l_{2} l_{4}} \tan \left((1 / 2) \sqrt{l_{2}} \xi\right)+l_{3}}+\left(\frac{\left(a_{1}(-\sqrt{\kappa}) l_{3}-4 i \sqrt{2} l_{4}\right)}{\sqrt{\kappa} l_{3}} \times\left[\frac{l_{4} l_{2}^{4} \sec ^{8}\left(\sqrt{l_{2}} \xi / 2\right)}{\left(2 \sqrt{l_{2} l_{4}} \tan \left((1 / 2) \sqrt{l_{2}} \xi\right)+l_{3}\right)^{4}}\right.\right.\right. \\
& \quad+\frac{l_{3} l_{2}^{3} \sec ^{6}\left(\sqrt{l_{2}} \xi / 2\right)}{\left(2 \sqrt{l_{2} l_{4}} \tan \left((1 / 2) \sqrt{l_{2}} \xi\right)+l_{3}\right)^{3}} \\
& \left.\left.\left.+\frac{l_{2}^{3} \sec ^{4}\left(\sqrt{l_{2}} \xi / 2\right)}{\left(2 \sqrt{l_{2} l_{4}} \tan \left((1 / 2) \sqrt{l_{2}} \xi\right)+l_{3}\right)^{2}}\right]^{1 / 2}\right)\right] \\
& \quad \times \exp \left[i \left(k_{1} x\right.\right. \\
& \left.\left.\quad+k_{2} y+\frac{1}{2} t\left(k_{1}^{2}-k_{2}^{2}\right)\right)\right] \\
& Q_{52}(\xi)=\frac{2 v}{3\left(1-\alpha^{2}\right)}\left(Z_{52} 3(\xi)\right)^{3} \\
& l_{2}>0 .
\end{aligned}
$$

\section{Modulation Instability of Davey-Stewartson Equation}

Equation (1) reveals a certain formal connection with the defocusing nonlinear Schrödinger equation in $1+1$ dimension $[51,52]$, given below

$$
i \frac{\partial Z}{\partial t}+\frac{1}{2} \alpha^{2} \frac{\partial^{2} Z}{\partial x^{2}}-v|Z|^{2} Z=0 .
$$

Most nonlinear higher-order NLPDEs demonstrate instability leading to the research of steady-state modulation as a result of interaction between nonlinear and dispersive impacts. The modulation instability analysis of (51) is carried out by using the standard linear stability analysis $[53,54]$ to check how weak and time dependent perturbations develop along the propagation distance $[53,54]$. The $1+1$ dimension defocusing nonlinear Schrödinger equation (51) of the Nonlinear (2+1)-Dimensional Davey-Stewartson Equation (1) has a state solution of the form

$$
\begin{aligned}
Z(x, y, t) & =\left(\sqrt{P}+\phi(x, y, t) e^{i \gamma}\right), \\
\gamma(t) & =P \beta t,
\end{aligned}
$$

where the optical power $\mathrm{P}$ is normalized and $\beta$ is a constant. The perturbation $\phi(x, y, t)$ is examined by utilizing linear stability analysis. Putting (52) into (51) and linearizing, we obtain

$$
i \frac{\partial \phi}{\partial t}+\frac{1}{2} \alpha^{2} \frac{\partial^{2} \phi}{\partial x^{2}}+\sqrt{P^{3}}(\beta+\nu)-3 P \nu \phi^{*}=0,
$$

where $*$ is a complex conjugate. The solution of (53) is considered in the form giving below

$$
\phi(x, y, t)=\psi_{1} e^{i\left(h_{1} x+h_{2} y-t \lambda\right)}+\psi_{2} e^{-i\left(h_{1} x+h_{2} y-t \lambda\right)},
$$

where $\lambda$ is the frequency of perturbation and $h_{1}, h_{2}$ normalized wave numbers. The dispersion relation determines how spatial oscillations $e^{i h_{1} x}, e^{i h_{2} y}$ are linked to time oscillations $e^{i \lambda t}$ of a wave number; substituting (54) into (53), we obtain the dispersive relation as

$$
\lambda= \pm \frac{\alpha^{2} \psi_{1} h_{1}^{2}+6 \psi_{2} \nu P}{2 \psi_{1}}, \quad \psi_{1} \neq 0 .
$$

The dispersion relation in (55) above demonstrates that the steady-state stability depends on the self-phase modulation, stimulated scattering, group velocity dispersion, and selfphase modulation. The expression of (55) shows that the value of the frequency $\lambda$ is real for all values of $h$ except when $\psi=0$, which means that the steady-state solution is stable.

\section{Results and Discussion}

Rational, periodic, and solitonic solutions for the DSE have been successfully obtained using the GEERE method. The constructed solutions by this method are novel and unique from other solutions obtained using different methods [4850]. Different forms of solutions such as trigonometric, hyperbolic trigonometric, and rational functions are obtained because the assumed solution of GEERE is different from other methods. Physical properties of some obtained results are demonstrated graphically using suitable parameters. Figure 1 evaluates the rational solitary wave solution (11), periodic solitary wave solution (13), and soliton solitary wave solution (15) plotted at $\left(a_{1}=1 ; \alpha=0.6 ; l_{0}=0.7 ; l_{1}=9 ; l_{2}=\right.$ $20 ; \kappa=0.7 ; c=0.1 ; y=0.8),\left(a_{1}=1 ; \alpha=0.6 ; l_{0}=0 ; l_{1}=\right.$ $\left.9 ; l_{2}=-30 ; \kappa=-0.7 ; c=0.1 ; y=0.8\right)$, and $\left(a_{1}=1 ; \alpha=\right.$ $\left.0.6 ; l_{0}=0.7 ; l_{1}=9 ; l_{2}=20 ; \kappa=0.7 ; c=0.1 ; y=0.8\right)$, respectively. The rational solitary wave solution (17), periodic solitary wave solution (19), and soliton solitary wave solution (21) are evaluated in Figure 2 plotted at $\left(a_{1}=1 ; \alpha=0.6 ; l_{0}=\right.$ $\left.0 ; l_{1}=9 ; l_{2}=20 ; \kappa=0.7 ; c=-0.1 ; y=0.8\right),\left(a_{1}=1 ; \alpha=\right.$ $\left.0.6 ; l_{0}=0 ; l_{1}=9 ; l_{2}=-30 ; \kappa=-0.7 ; c=-0.1 ; y=0.8\right)$, and $\left(a_{1}=1 ; \alpha=0.6 ; l_{0}=0.7 ; l_{1}=9 ; l_{2}=20 ; \kappa=0.7 ; c=\right.$ $-0.1 ; y=0.8)$, respectively. Figure 3 shows a periodic solitary wave solution (23) and a dark solitary wave solution (25) plotted at $\left(a_{1}=1 ; \alpha=0.6 ; l_{0}=0.7 ; l_{4}=9 ; l_{2}=30 ; \kappa=\right.$ $-0.7 ; c=0.1 ; y=0.8)$ and $\left(a_{1}=1 ; \alpha=0.6 ; l_{0}=0.7 ; l_{4}=\right.$ 


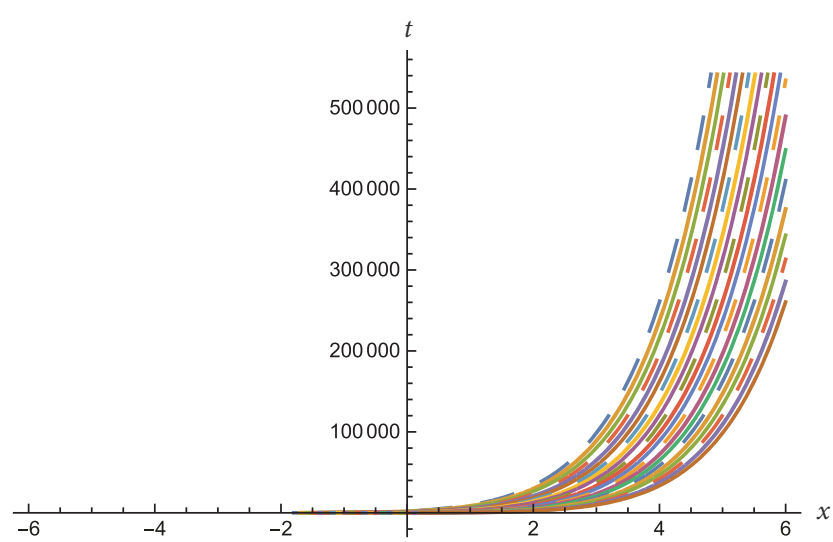

(a)

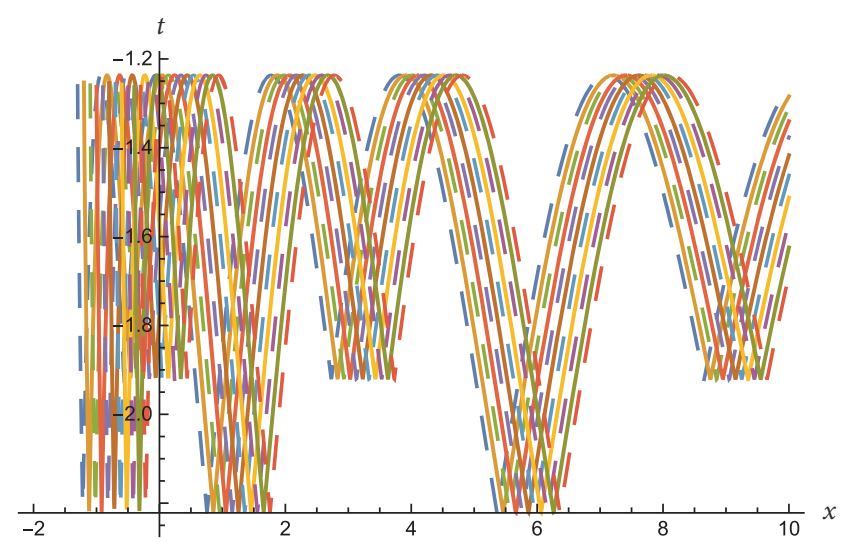

(b)

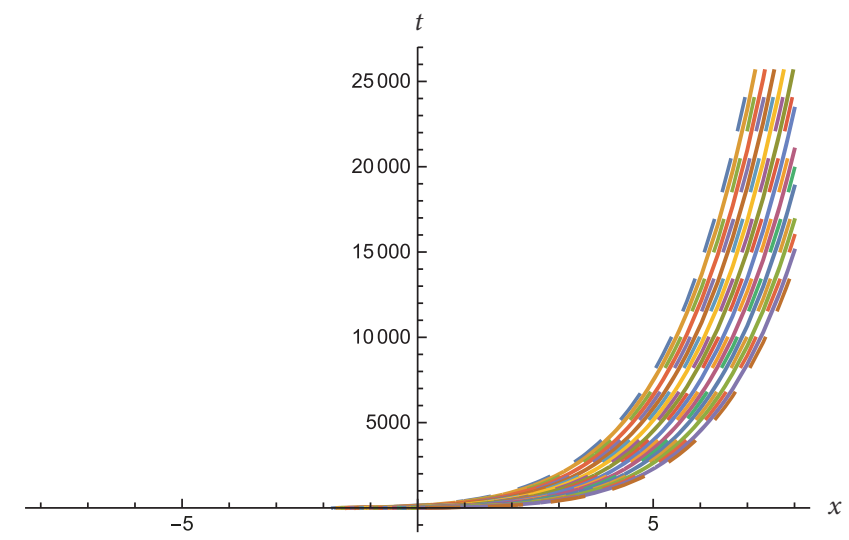

(c)

FIGURE 1: Plot of the exact traveling wave solutions of (a) rational solitary wave solution (11) with parameters $a_{1}=1 ; \alpha=0.6 ; l_{0}=0.7 ; l_{1}=$ $9 ; l_{2}=20 ; \kappa=0.7 ; c=0.1 ; y=0.8$; (b) periodic solitary solution (13) plotted at $a_{1}=1 ; \alpha=0.6 ; l_{0}=0 ; l_{1}=9 ; l_{2}=-30 ; \kappa=-0.7 ; c=0.1 ; y=$ 0.8 ; and (c) soliton solitary solution (15) plotted at $a_{1}=1 ; \alpha=0.6 ; l_{0}=0.7 ; l_{1}=9 ; l_{2}=20 ; \kappa=0.7 ; c=0.1 ; y=0.8$.

$\left.9 ; l_{2}=-10 ; \kappa=0.7 ; c=0.1 ; y=0.8\right)$, respectively. Periodic solitary wave solution (27) and dark solitary wave solution (29) plotted at $\left(a_{1}=1 ; \alpha=0.6 ; l_{0}=0.7 ; l_{4}=9 ; l_{2}=30 ; \kappa=\right.$ $-0.7 ; c=0.1 ; y=0.8)$ and $\left(a_{1}=1 ; \alpha=0.6 ; l_{0}=0.7 ; l_{4}=\right.$ $\left.9 ; l_{2}=-10 ; \kappa=0.7 ; c=0.1 ; y=0.8\right)$, respectively, are evaluated in Figure 4. Figure 5 evaluates bright solitary wave solution (31) and soliton solitary wave solution (33) plotted at $\left(a_{1}=1 ; \alpha=0.6 ; l_{1}=9 ; l_{2}=-10 ; \kappa=0.7 ; c=0.1 ; y=0.8\right)$ and $\left(a_{1}=1 ; \alpha=0.6 ; l_{1}=9 ; l_{2}=10 ; \kappa=0.7 ; c=0.1 ; y=0.8\right)$, respectively. Periodic solitary wave solution (35) and soliton solitary wave solution (37) plotted at $\left(a_{1}=1 ; \alpha=0.6 ; l_{1}=\right.$ $\left.9 ; l_{2}=-10 ; \kappa=0.7 ; c=0.1 ; y=0.8\right)$ and $\left(a_{1}=9 ; \alpha=\right.$ $\left.0.6 ; l_{1}=9 ; l_{2}=10 ; \kappa=0.7 ; c=0.1 ; y=0.8\right)$, respectively, are evaluated in Figure 6. Figure 7 shows periodic solitary wave solution (39) and dark solitary wave solution (41) plotted at $\left(a_{1}=9 ; \alpha=0.6 ; l_{2}=-1 ; l_{3}=-1 ; \kappa=0.7 ; c=0.1 ; y=0.8\right)$ and $\left(a_{1}=9 ; \alpha=0.6 ; l_{2}=9 ; l_{3}=1 ; \kappa=0.7 ; c=0.1 ; y=0.8\right)$, respectively. Figure 8 shows periodic solitary wave solution (43) and dark solitary wave solution (45) plotted at $\left(a_{1}=\right.$ $\left.9 ; \alpha=0.6 ; l_{2}=-1 ; l_{3}=-1 ; \kappa=0.7 ; c=0.1 ; y=0.8\right)$ and $\left(a_{1}=1 ; \alpha=0.6 ; l_{2}=1 ; l_{3}=1 ; \kappa=0.7 ; c=0.1 ; y=0.8\right)$, respectively. Also periodic solitary wave solution (47) and dark solitary wave solution (49) plotted at $\left(a_{1}=9 ; l_{2}=\right.$ $\left.-3 ; l_{3}=-3 ; l_{4}=1 ; \kappa=0.7 ; c=0.1 ; y=0.8\right)$ and $\left(a_{1}=1 ; l_{2}=5 ; l_{3}=5 ; l_{4}=0 ; \kappa=0.7 ; c=0.1 ; y=0.8\right)$, respectively, are evaluated in Figure 9. The dispersion relation among frequency $(\lambda)$ and wave numbers $\left(h_{1}, h_{2}\right)$ of $(55)$ is plotted in Figure 10. This relation determines how spatial oscillations $e^{i h_{2} x}, e^{i h_{2} y}$ are linked to time oscillations $e^{i \lambda t}$ of a wave number of obtained solutions for the DSE.

\section{Conclusion}

By implementing the strong GEERE technique, this article has effectively built fresh solitonic and reasonable regular solutions for the DSE. Some freshly obtained solitary wave solutions are provided graphically to display their physical characteristics and also provide information on the circumstances for the creation of solitons of light and kink. The solutions extracted have theoretical and experimental applications that can be anticipated to occur in other associated revolutionary equations such as the nonlinear Schrödinger equation to explain the Bose-Einstein condensation. We contrasted our solution to current existing solution and claimed that there are many new solutions. The equation recognize the huge variety of possible solutions for only values of a small subset of parameters, which helps to comprehend the physical phenomena of this equation. Modulation instability 


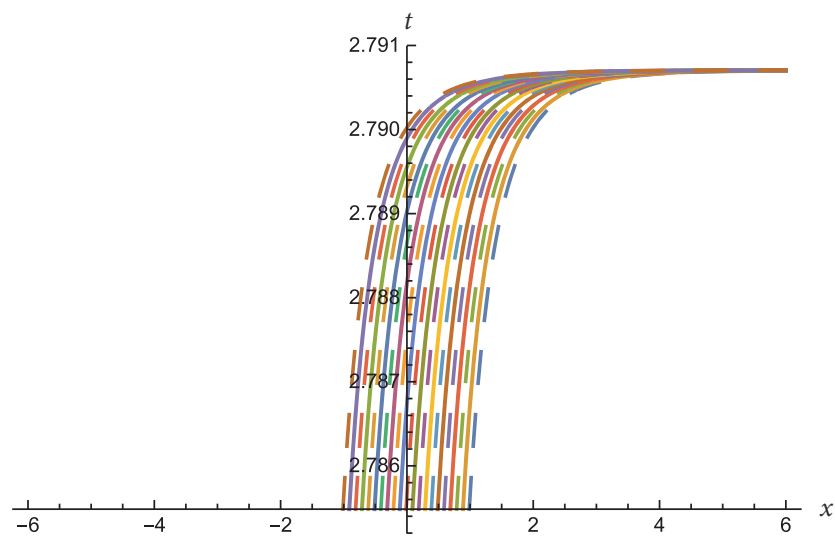

(a)

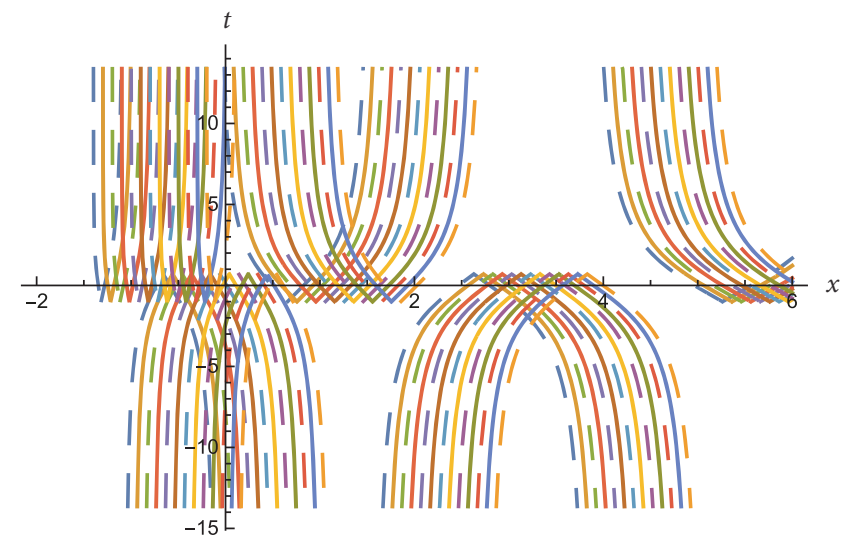

(b)

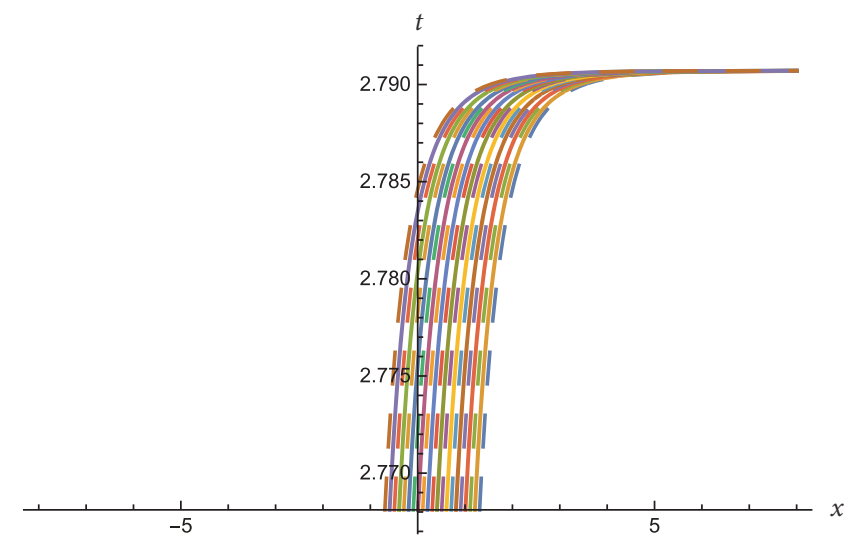

(c)

Figure 2: Plot of the exact traveling wave solutions of (a) rational solitary wave solution (17) with parameters $a_{1}=1 ; \alpha=0.6 ; l_{0}=0 ; l_{1}=$ $9 ; l_{2}=20 ; \kappa=0.7 ; c=-0.1 ; y=0.8$; (b) periodic solitary wave solution (19) plotted at $a_{1}=1 ; \alpha=0.6 ; l_{0}=0 ; l_{1}=9 ; l_{2}=-30 ; \kappa=-0.7 ; c=$ $-0.1 ; y=0.8$; and (c) soliton solitary wave solution (21) plotted at $a_{1}=1 ; \alpha=0.6 ; l_{0}=0.7 ; l_{1}=9 ; l_{2}=20 ; \kappa=0.7 ; c=-0.1 ; y=0.8$.

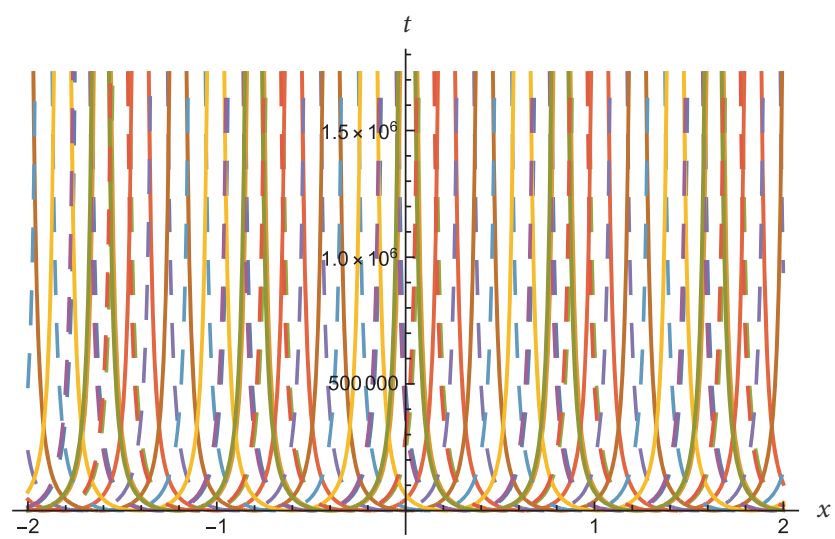

(a)

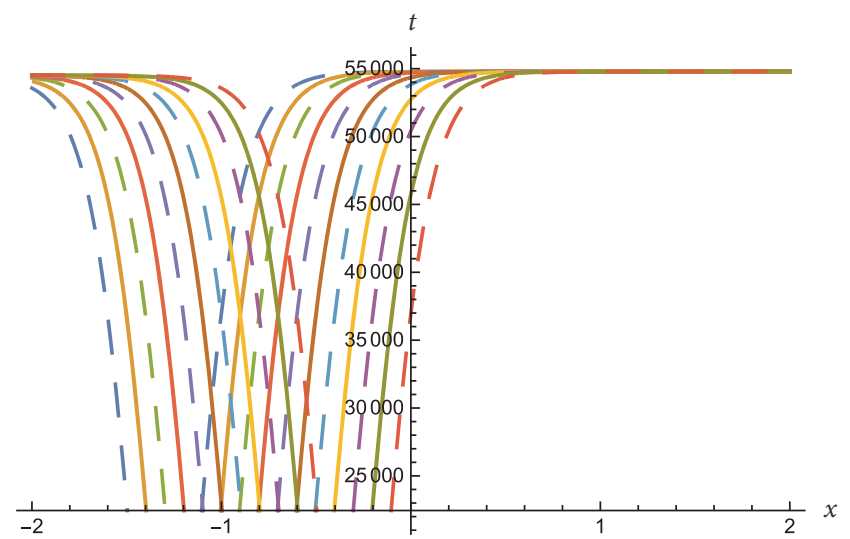

(b)

FIGURE 3: Plot of the exact traveling wave solutions of (a) periodic solitary wave solution (23) with parameters $a_{1}=1 ; \alpha=0.6 ; l_{0}=0.7 ; l_{4}=$ $9 ; l_{2}=20 ; \kappa=0.7 ; c=0.1 ; y=0.8$; (b) dark solitary wave solution (25) plotted at $a_{1}=1 ; \alpha=0.6 ; l_{0}=0 ; l_{4}=9 ; l_{2}=-30 ; \kappa=0.7 ; c=0.1 ; y=$ 0.8 . 


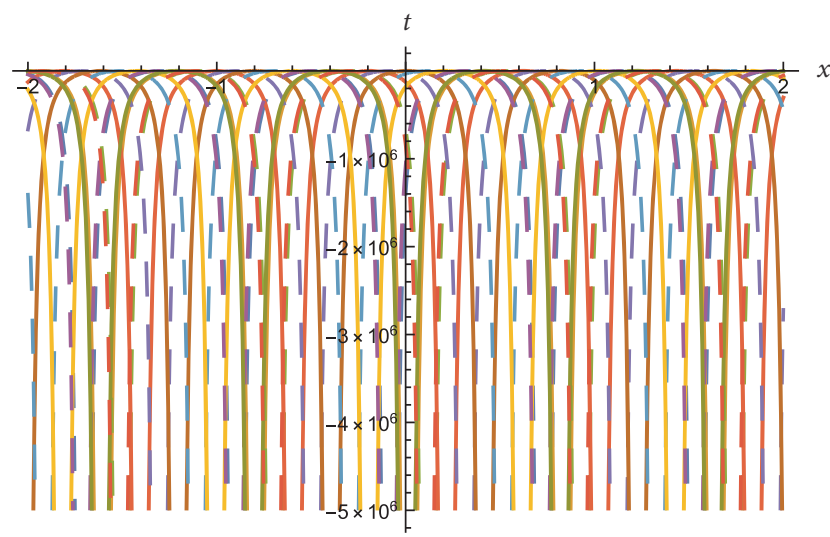

(a)

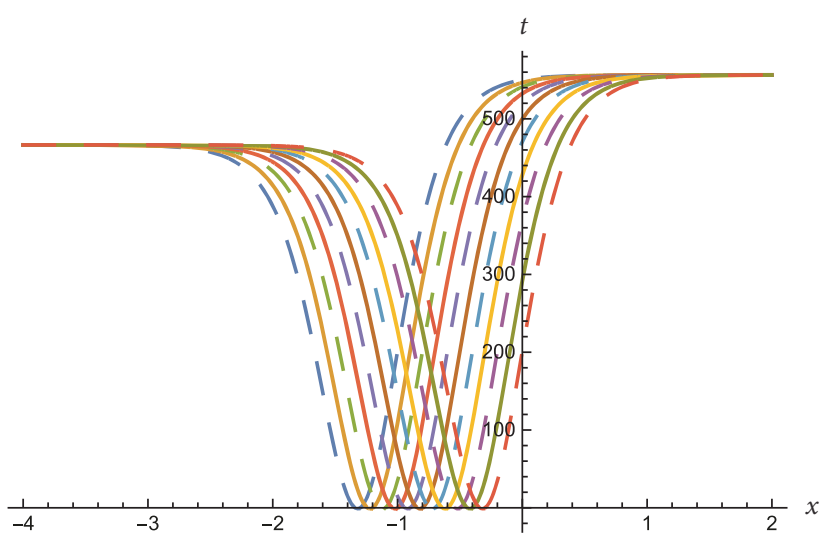

(b)

FIGURE 4: Plot of the exact traveling wave solutions of (a) periodic solitary wave solution (27) with parameters $a_{1}=1 ; \alpha=0.6 ; l_{0}=0.7 ; l_{4}=$ $9 ; l_{2}=30 ; \kappa=-0.7 ; c=0.1 ; y=0.8$; (b) dark solitary wave solution (29) plotted at $a_{1}=1 ; \alpha=0.6 ; l_{0}=0.7 ; l_{4}=9 ; l_{2}=-10 ; \kappa=0.7 ; c=$ $0.1 ; y=0.8$.

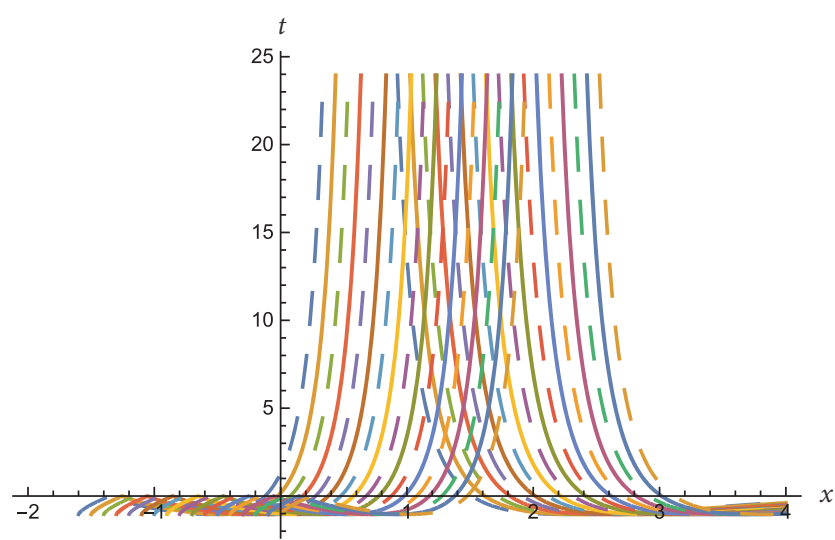

(a)

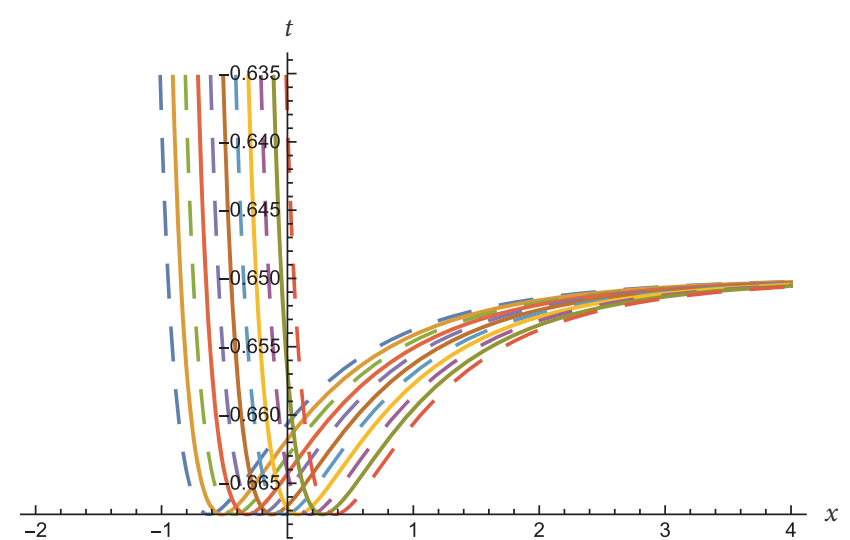

(b)

Figure 5: Plot of the exact traveling wave solutions of (a) bright solitary wave solution (31) with parameters $a_{1}=1 ; \alpha=0.6 ; l_{1}=9 ; l_{2}=$ $-10 ; \kappa=0.7 ; c=0.1 ; y=0.8$; (b) soliton solitary wave solution (33) plotted at $a_{1}=1 ; \alpha=0.6 ; l_{1}=9 ; l_{2}=10 ; \kappa=0.7 ; c=0.1 ; y=0.8$.

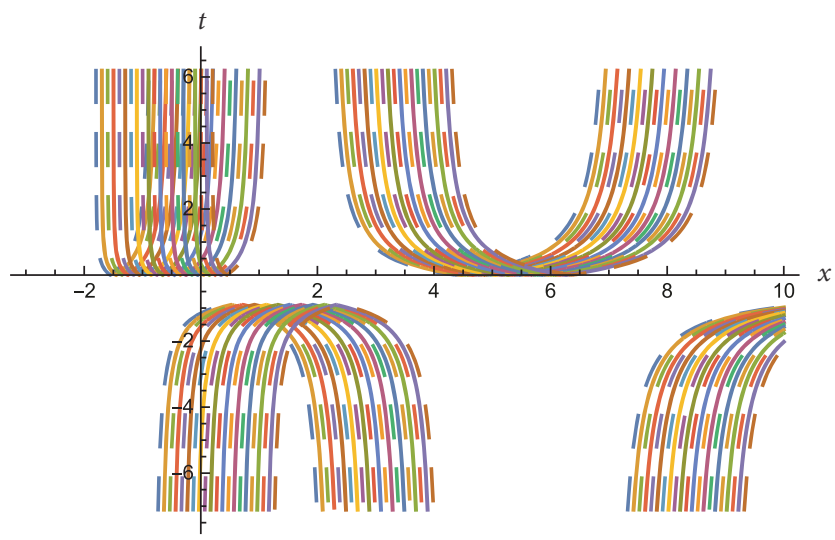

(a)

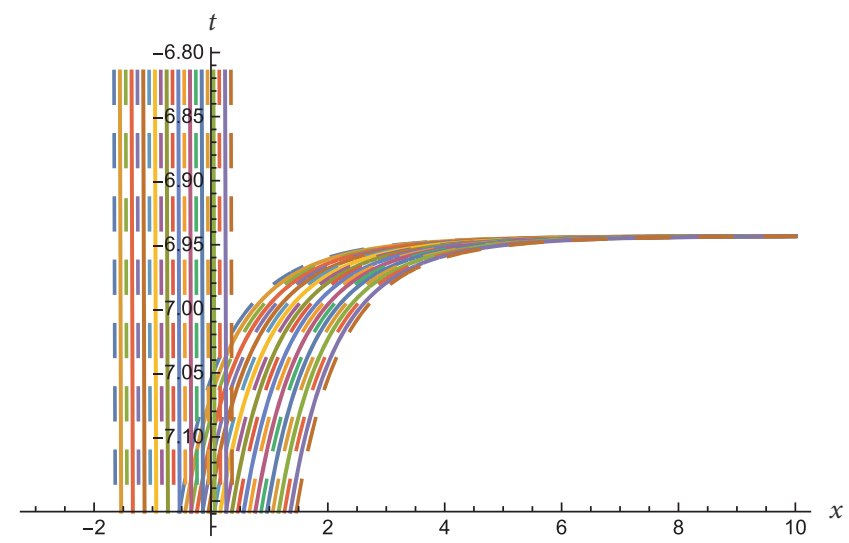

(b)

FIGURE 6: Plot of the exact traveling wave solutions of (a) periodic solitary wave solution (35) with parameters $a_{1}=1 ; \alpha=0.6 ; l_{1}=9 ; l_{2}=$ $-10 ; \kappa=0.7 ; c=0.1 ; y=0.8$; (b) soliton solitary wave solution (37) plotted at $a_{1}=9 ; \alpha=0.6 ; l_{1}=9 ; l_{2}=10 ; \kappa=0.7 ; c=0.1 ; y=0.8$. 


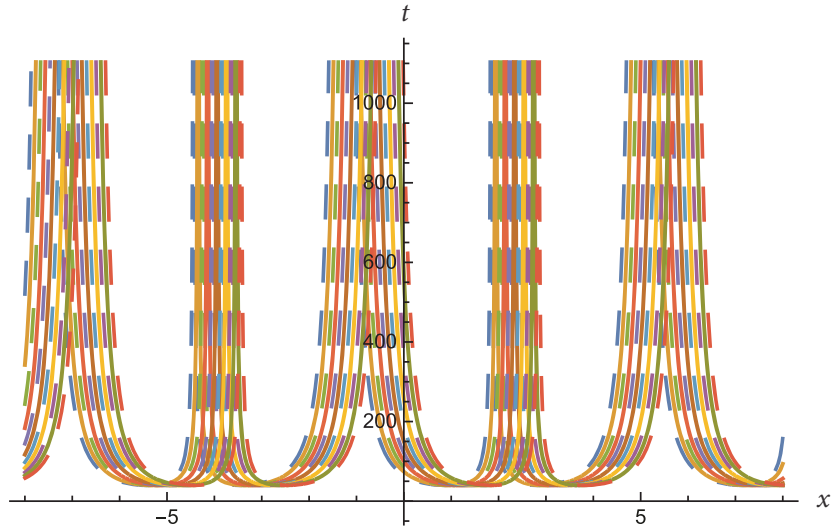

(a)

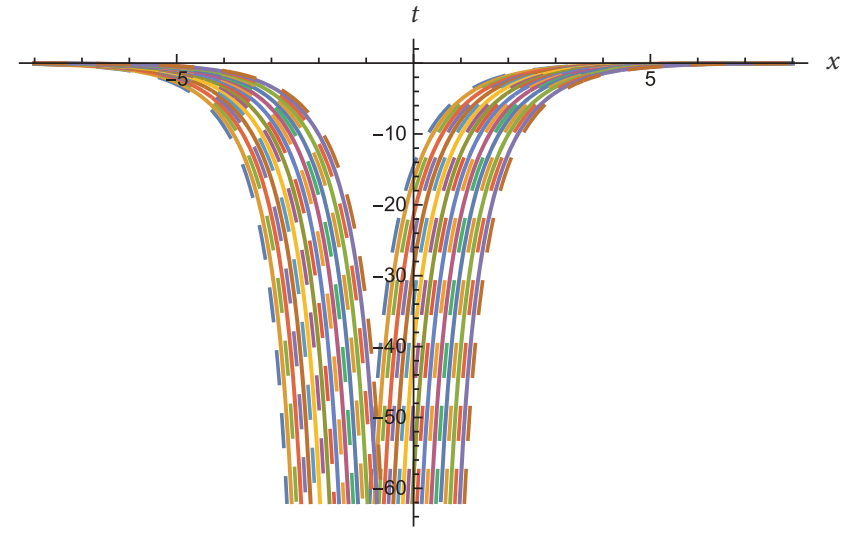

(b)

FIGURE 7: Plot of the exact traveling wave solutions of (a) periodic solitary wave solution (39) with parameters $a_{1}=9 ; \alpha=0.6 ; l_{2}=-1 ; l_{3}=$ $-1 ; \kappa=0.7 ; c=0.1 ; y=0.8$; (b) dark solitary wave solution (41) plotted at $a_{1}=9 ; \alpha=0.6 ; l_{2}=9 ; l_{3}=1 ; \kappa=0.7 ; c=0.1 ; y=0.8$.

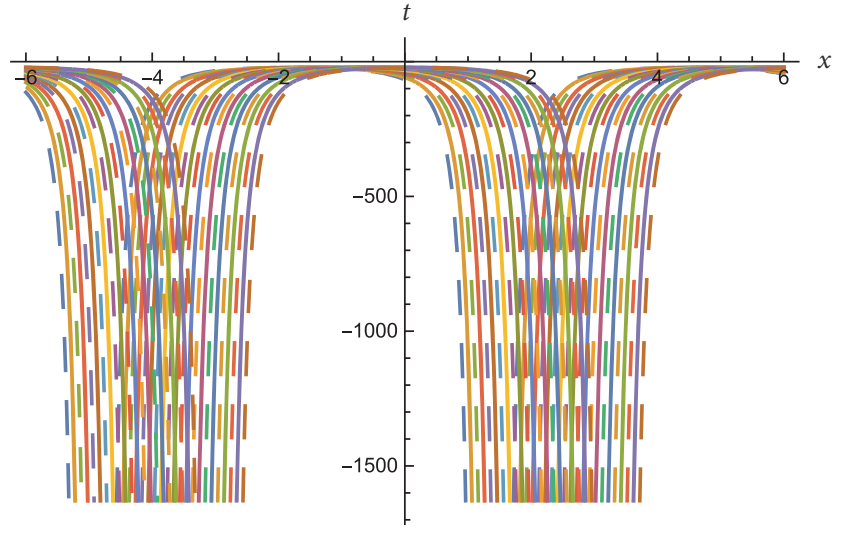

(a)

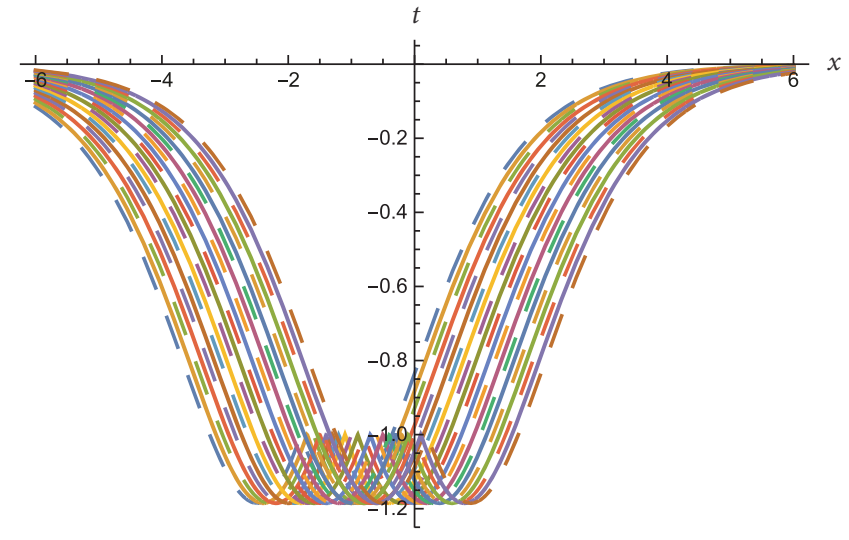

(b)

FIGURE 8: Plot of the exact traveling wave solutions of (a) periodic solitary wave solution (43) with parameters $a_{1}=9 ; \alpha=0.6 ; l_{2}=-1 ; l_{3}=$ $-1 ; \kappa=0.7 ; c=0.1 ; y=0.8$; (b) dark solitary wave solution (45) plotted at $a_{1}=1 ; \alpha=0.6 ; l_{2}=1 ; l_{3}=1 ; \kappa=0.7 ; c=0.1 ; y=0.8$.

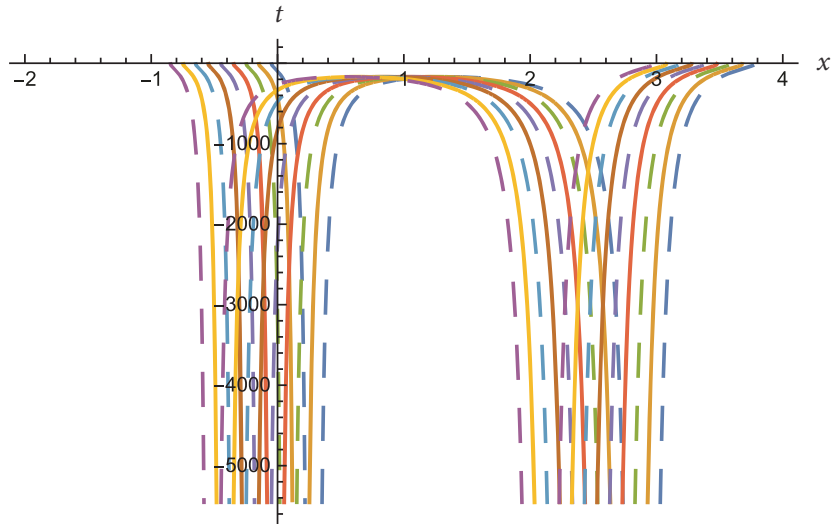

(a)

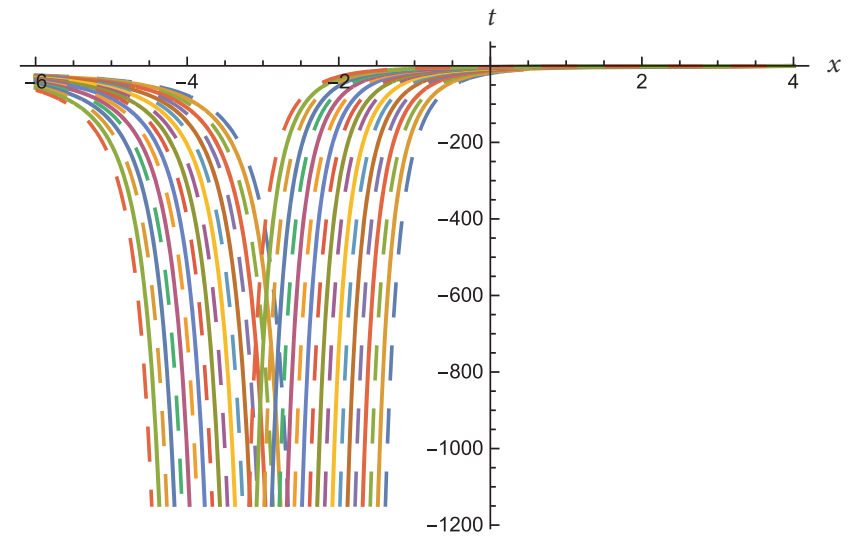

(b)

FIGURE 9: Plot of the exact traveling wave solutions of (a) periodic solitary wave solution (47) with parameters $a_{1}=9 ; l_{2}=-3 ; l_{3}=-3 ; l_{4}=$ $1 ; \kappa=0.7 ; c=0.1 ; y=0.8$; (b) dark solitary wave solution (49) plotted at $a_{1}=1 ; l_{2}=5 ; l_{3}=5 ; l_{4}=0 ; \kappa=0.7 ; c=0.1 ; y=0.8$. 


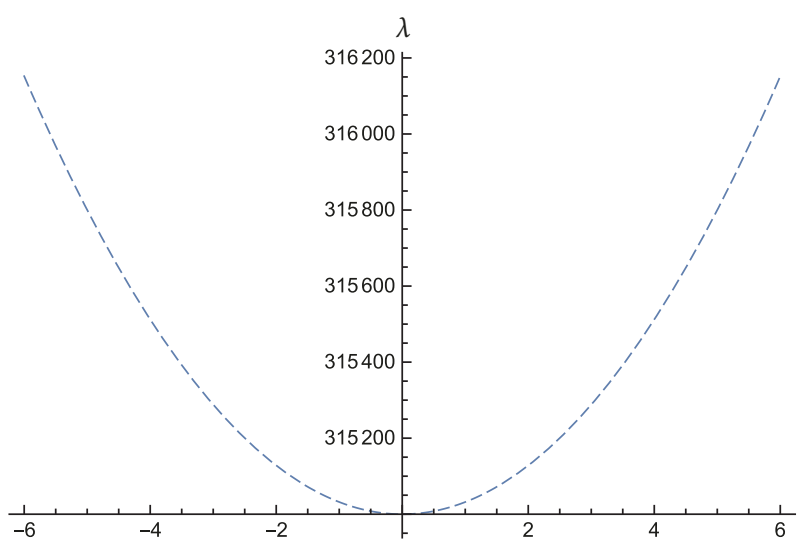

Figure 10: Diagram of dispersion relation among frequency $(\lambda)$ and wave number $\left(h_{1}\right)$.

assessment is used to verify the stability of the solutions acquired. We conclude that the modulation instability analytical expression is gained, which demonstrates that all built solutions are accurate and stable. The effectiveness and simplicity of the suggested GEERE technique show that it can be applied to distinct kinds of separate nonlinear models in different nonlinear science fields.

\section{Data Availability}

This manuscript is purely mathematical physics, and all computations were done using Mathematica software and did not involve any data.

\section{Conflicts of Interest}

The authors declare that they have no conflicts of interest.

\section{References}

[1] A. R. Seadawy, "Stability analysis for Zakharov-Kuznetsov equation of weakly nonlinear ion-acoustic waves in a plasma," Computers \& Mathematics with Applications, vol. 67, no. 1, pp. 172-180, 2014.

[2] S. F. Tian, "Initial-boundary value problems for the general coupled nonlinear schrodinger equation on the interval via thefokas method," Journal of Differential Equations, vol. 262, pp. 506-558, 2017.

[3] J.-H. He, "Homotopy perturbation method for bifurcation of nonlinear problems," International Journal of Nonlinear Sciences and Numerical Simulation, vol. 6, no. 2, pp. 207-208, 2005.

[4] A. R. Seadawy, "Stability analysis solutions for nonlinear three-dimensional modified Korteweg-de Vries-ZakharovKuznetsov equation in a magnetized electron-positron plasma," Physica A: Statistical Mechanics and its Applications, vol. 455, pp. 44-51, 2016.

[5] Q. M. Al-Mdallal, "A new family of exact solutions to the unsteady Navier-Stokes equations using canonical transformation with complex coefficients," Applied Mathematics and Computation, vol. 196, no. 1, pp. 303-308, 2008.
[6] Q. M. Al-Mdallal and M. I. Syam, "Sine-Cosine method for finding the soliton solutions of the generalized fifth-order nonlinear equation," Chaos, Solitons \& Fractals, vol. 33, no. 5, pp. 1610-1617, 2007.

[7] A. V. Porubov and M. G. Velarde, "Exact periodic solutions of the complex Ginzburg-Landau equation," Journal of Mathematical Physics, vol. 40, no. 2, pp. 884-896, 1999.

[8] A. Huber, "The calculation of novel class of solutions of a non-linear fourth order evolution equation by the Weierstrass transform method," Applied Mathematics and Computation, vol. 201, no. 1-2, pp. 668-677, 2008.

[9] A. Huber, "A novel class of solutions for a non-linear third order wave equation generated by the Weierstrass transformation," Chaos, Solitons \& Fractals, vol. 28, no. 4, pp. 972-978, 2006.

[10] E. A. Wakil, E. M. Abulwafa, and M. A. Abdou, "Extended Weierstrass transformation method for nonlinear evolution equations," Nonlinear Science Letters A: Mathematics, Physics and Mechanics, vol. 1, no. 3, pp. 253-262, 2010.

[11] M. J. Wang and Q. Wang, "Application of rational expansion method for stochastic differential equations," Applied Mathematics and Computation, vol. 218, no. 9, pp. 5259-5264, 2012.

[12] A. Syam Kumar, J. Prasanth, and V. M. Bannur, "Quarkgluon plasma phase transition using cluster expansion method," Physica A: Statistical Mechanics and its Applications, vol. 432, pp. 71-75, 2015.

[13] G. Chaohao, Soliton Theory and Its Applications, Zhejiang Science and Technology Press, 1990.

[14] M. J. Ablowitz and P. A. Clarkson, Solitons, Nonlinear Evolution Equations and Inverse Scattering, Cambridge University Press, New York, NY, USA, 1991.

[15] Abdullah, A. R. Seadawy, and W. Jun, "Mathematical methods and solitary wave solutions of three-dimensional ZakharovKuznetsov-Burgers equation in dusty plasma and its applications," Results in Physics, vol. 7, pp. 4269-4277, 2017.

[16] Q. Zhao and L. Wu, "Darboux transformation and explicit solutions to the generalized TD equation," Applied Mathematics Letters, vol. 67, pp. 1-6, 2017.

[17] A. R. Seadawy, "Stability analysis for two-dimensional ionacoustic waves in quantum plasmas," Physics of Plasmas, vol. 21, no. 5, Article ID 052107, 2014.

[18] R. Hirota, "Exact $N$-soliton solutions of the wave equation of long waves in shallow-water and in nonlinear lattices," Journal of Mathematical Physics, vol. 14, pp. 810-814, 1973.

[19] M. Arshad, A. Seadawy, D. Lu, and J. Wang, "Travelling wave solutions of Drinfel'd-Sokolov-Wilson, Whitham-Broer-Kaup and (2+1)-dimensional Broer-Kaup-Kupershmit equations and their applications," Chinese Journal of Physics, vol. 55, no. 3, pp. 780-797, 2017.

[20] A. Esen, "A numerical solution of the equal width wave equation by a lumped Galerkin method," Applied Mathematics and Computation, vol. 168, no. 1, pp. 270-282, 2005.

[21] K. U. Tariq and A. Seadawy, "Bistable Bright-Dark solitary wave solutions of the $(3+1)$-dimensional Breaking soliton, Boussinesq equation with dual dispersion and modified Korteweg-de Vries-Kadomtsev-Petviashvili equations and their applications," Results in Physics, vol. 7, pp. 1143-1149, 2017.

[22] T. A. Sulaiman, A. Yokus, N. Gulluoglu et al., "Regarding the numerical solutions of the Sharma-Tasso-Olver equation," in Proceedings of the 3rd International Conference on Computational Mathematics and Engineering Sciences- (CMES- 2018), vol. 22, Girne, Cyprus, 2018. 
[23] C. Cattani, T. A. Sulaiman, H. M. Baskonus, and H. Bulut, "Solitons in an inhomogeneous Murnaghan's rod," The European Physical Journal Plus, vol. 133, no. 228, pp. 1-12, 2018.

[24] H. M. Baskonus, "New complex and hyperbolic function solutions to the generalized double combined Sinh-Cosh-Gordon equation," AIP Conference Proceedings 1798, Article ID 020018, pp. 1-9, 2017.

[25] H. M. Baskonus, "New acoustic wave behaviors to the DaveyStewartson equation with power-law nonlinearity arising in fluid dynamics," Nonlinear Dynamics, vol. 86, no. 1, pp. 177-183, 2016.

[26] L. I. Aliyu, M. Inc, A. Yusuf, and D. Baleanu, "Symmetry analysis, explicit solutions, and conservation laws of a sixthorder nonlinear ramani equation," Symmetry, vol. 10, no. 8, p. 341, 2018.

[27] M. Inc, M. Hashemi, and A. Isa Aliyu, "Exact solutions and conservation laws of the bogoyavlenskii equation," Acta Physica Polonica A, vol. 133, no. 5, pp. 1133-1137, 2018.

[28] A. I. Aliyu, F. Tchier, M. Inc, A. Yusuf, and D. Baleanu, "Dynamics of optical solitons, multipliers and conservation laws to the nonlinear schrodinger equation in $(2+1)$-dimensions with non-Kerr law nonlinearity," Journal of Modern Optics, vol. 66, no. 2, pp. 136-142, 2019.

[29] M. Inc, A. I. Aliyu, A. Yusuf, D. Baleanu, and E. Nuray, "Complexiton and solitary wave solutions of the coupled nonlinear Maccari's system using two integration schemes," Modern Physics Letters B, vol. 32, no. 02, p. 1850014, 2018.

[30] A. I. Aliyu, M. Inc, A. Yusuf, and D. Baleanu, "Optical solitons and stability analysis in ring-cavity fiber system with carbon nanotube as saturable absorber," Communications in Theoretical Physics, vol. 70, no. 5, p. 511, 2018.

[31] M. Inc, A. I. Aliyu, A. Yusuf, and D. Baleanu, "Optical and singular solitary waves to the PNLSE with third order dispersion in Kerr media via two integration approaches," Optik International Journal for Light and Electron Optics, vol. 163, pp. 142-151, 2018.

[32] A. Davey and K. Stewartson, "On three-dimensional packets of surface waves," Proceedings of the Royal Society A Mathematical, Physical and Engineering Sciences, vol. 338, no. 1613, pp. 101-110, 1974.

[33] C. Babaoglu, "Long-wave short-wave resonance case for a generalized Davey-Stewartson system," Chaos, Solitons \& Fractals, vol. 38, no. 1, pp. 48-54, 2008.

[34] K. W. Chow and S. Y. Lou, "Propagating wave patterns and "peakons" of the Davey-Stewartson system," Chaos, Solitons \& Fractals, vol. 27, no. 2, pp. 561-567, 2006.

[35] G. Ebadi, E. Krishnan, M. Labidi, E. Zerrad, and A. Biswas, "Analytical and numerical solutions to the Davey-Stewartson equation with power-law nonlinearity," Waves in Random and Complex Media, vol. 21, no. 4, pp. 559-590, 2011.

[36] G. Ebadi and A. Biswas, "The G/G method and 1-soliton solution of the Davey-Stewartson equation," Mathematical and Computer Modelling, vol. 53, no. 5-6, pp. 694-698, 2011.

[37] A. Bekir and A. C. Cevikel, "New solitons and periodic solutions for nonlinear physical models in mathematical physics," Nonlinear Analysis: Real World Applications, vol. 11, no. 4, pp. 3275-3285, 2010

[38] X. Zhao, "Self-similar solutions to a generalized Davey-Stewartson system," Mathematical and Computer Modelling, vol. 50, no. 9-10, pp. 1394-1399, 2009.
[39] M. Tajiri and T. Arai, "Growing-and-decaying mode solution to the Davey-Stewartson equation," Physical Review E: Statistical, Nonlinear, and Soft Matter Physics, vol. 60, no. 2, pp. 2297-2305, 1999.

[40] M. Tajiri, K. Takeuchi, and T. Arai, "Asynchronous development of the benjamin-feir unstable mode: solution of the daveystewartson equation," Physical Review E, vol. 64, p. 56622, 2001.

[41] M. Tajiri, H. Miura, and T. Arai, "Resonant interaction of modulational instability with a periodic soliton in the DaveyStewartson equation," Physical Review E: Statistical, Nonlinear, and Soft Matter Physics, vol. 66, no. 6, Article ID 67601, 2002.

[42] X. Y. Tang, K. W. Chow, and C. Rogers, "Propagating wave patterns for the 'resonant' Davey-Stewartson system," Chaos, Solitons \& Fractals, vol. 42, no. 5, pp. 2707-2712, 2009.

[43] Z. Khan, M. Qasim, R. U. Haq, and Q. M. Al-Mdallal, "Closed form dual nature solutions of fluid flow and heat transfer over a stretching/shrinking sheet in a porous medium," Chinese Journal of Physics, vol. 55, no. 4, pp. 1284-1293, 2017.

[44] Y. Wan, L. Song, L. Yin, and H. Zhang, "Generalized method and new exact wave solutions for $(2+1)$-dimensional Broer-Kaup-Kupershmidt system," Applied Mathematics and Computation, vol. 187, no. 2, pp. 644-657, 2007.

[45] I. Kourakis and P. K. Shukla, "Modulated wavepackets associated with longitudinal dust grain oscillations in a dusty plasma crystal," Physics of Plasmas, vol. 11, no. 4, pp. 1384-1393, 2004.

[46] A. R. Seadawy, M. Arshad, and D. Lu, "Stability analysis of new exact traveling-wave solutions of new coupled KdV and new coupled Zakharov-Kuznetsov systems," The European Physical Journal Plus, vol. 132, no. 162, pp. 1-19, 2017.

[47] A. R. Seadawy and D. Lu, "Ion acoustic solitary wave solutions of three-dimensional nonlinear extended Zakharov-Kuznetsov dynamical equation in a magnetized two-ion-temperature dusty plasma," Results in Physics, vol. 6, pp. 590-593, 2016.

[48] A. Davey and K. Stewartson, "On three-dimensional packets of surface waves," Proceedings of the Royal Society of London. Series A, vol. 338, pp. 101-110, 1974.

[49] H. A. Zedan and S. J. Monaquel, "The sine-cosine method for the davey-stewartson equations," Applied Mathematics E - Notes, vol. 10, pp. 103-111, 2010.

[50] H. Jafari, A. Kadem, D. Baleanu, and T. Yilmaz, "Solutions of the fractional davey-stewartson equations with variational iteration method," Romanian Reports in Physics, vol. 64, no. 2, pp. 337346, 2012.

[51] O. Assainova, C. Klein, K. D. McLaughlin, and P. D. Miller, "A study of the direct spectral transform for the defocusing daveystewartson II equation in the semiclassical limit," Communications on Pure and Applied Mathematics, vol. 72, no. 7, pp. 14741547, 2019.

[52] R.-J. Wang and Y.-C. Huang, "Exact solutions and excitations for the Davey-Stewartson equations with nonlinear and gain terms," The European Physical Journal D, vol. 57, no. 3, pp. 395401, 2010.

[53] M. Arshad, A. R. Seadawy, and D. Lu, "Exact bright-dark solitary wave solutions of the higher-order cubic-quintic nonlinear Schrödinger equation and its stability," Optik - International Journal for Light and Electron Optics, vol. 138, pp. 40-49, 2017.

[54] M. Arshad, A. R. Seadawy, D. Lu, and W. Jun, "Modulation instability analysis of modify unstable nonlinear schrodinger dynamical equation and its optical soliton solutions," Results in Physics, vol. 7, pp. 4153-4161, 2017. 


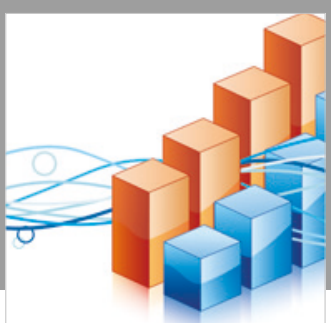

Advances in

Operations Research

\section{-n-m}
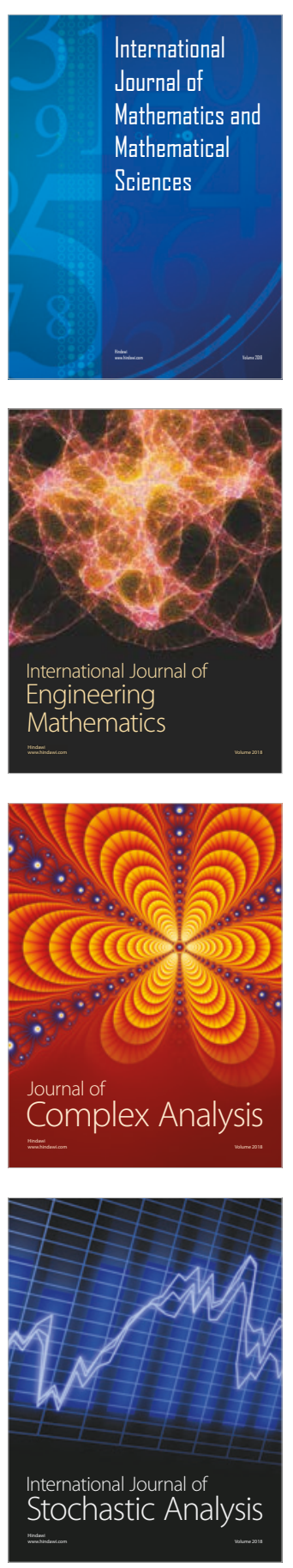
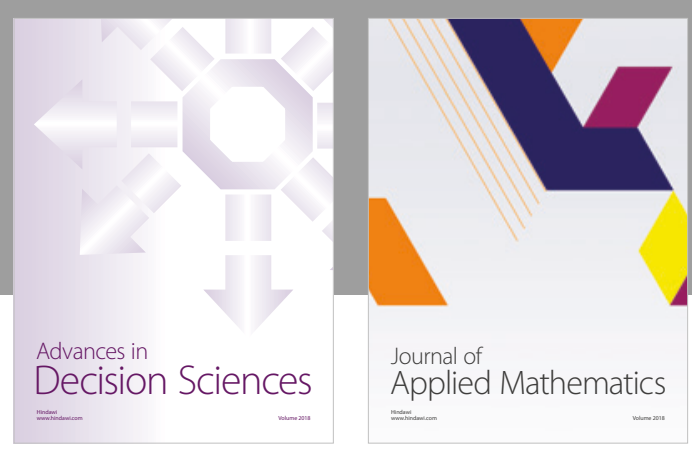

Journal of

Applied Mathematics
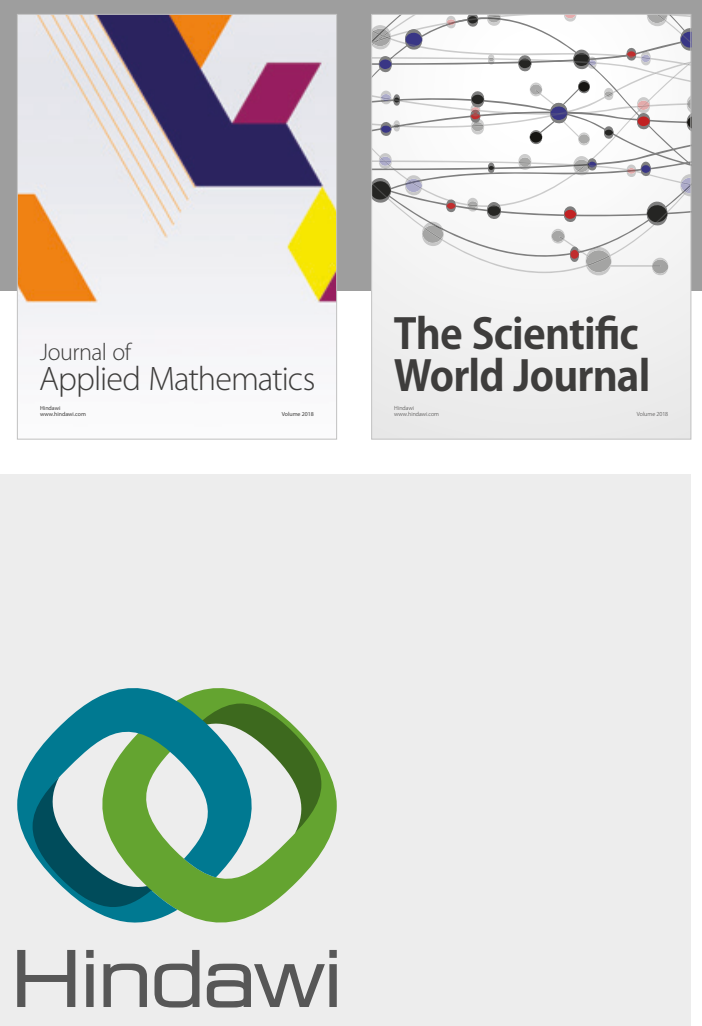

Submit your manuscripts at

www.hindawi.com

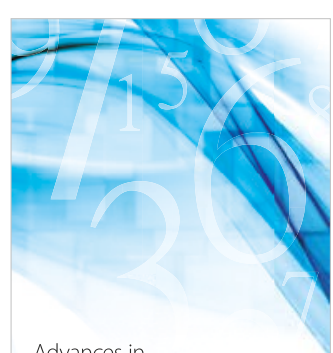

Advances in
Numerical Analysis
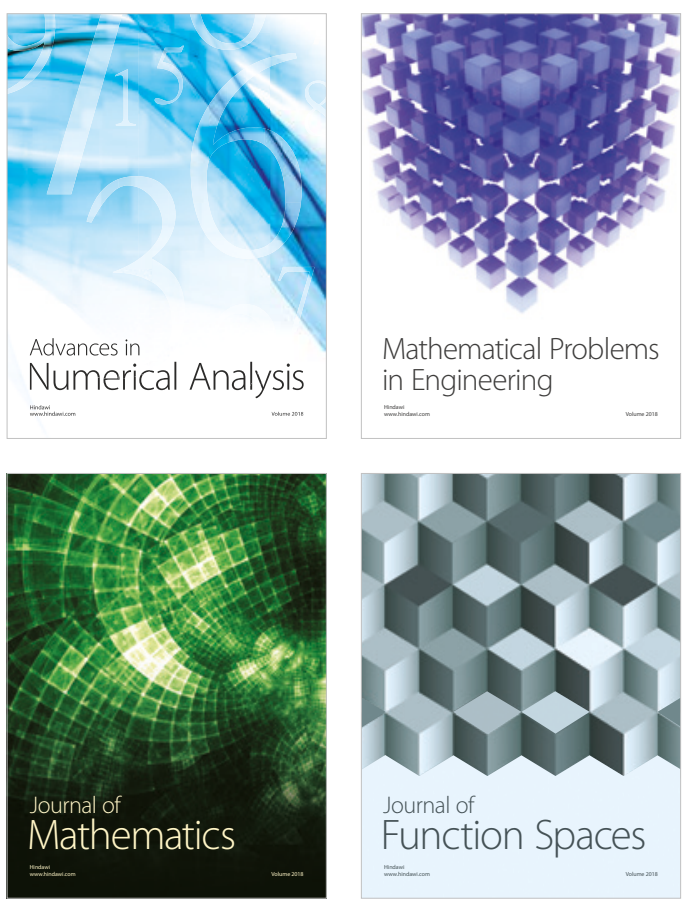

Mathematical Problems in Engineering

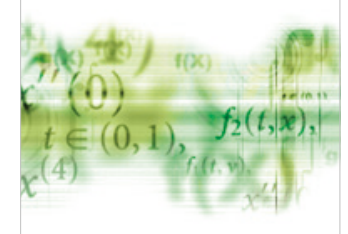

International Journal of

Differential Equations

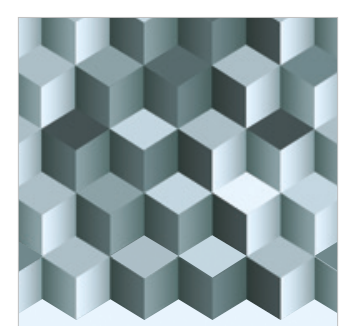

Journal of

Function Spaces

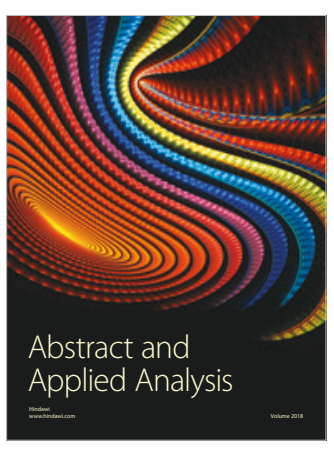

The Scientific

World Journal

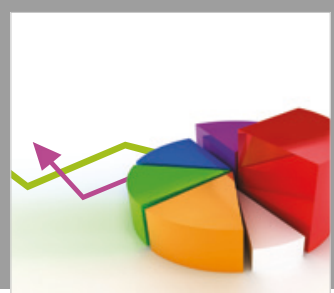

Journal of

Probability and Statistics
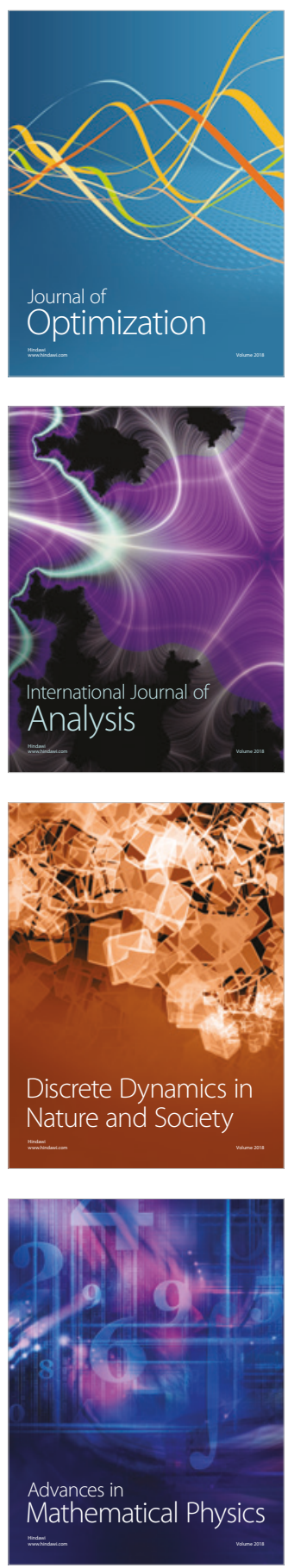